University of Windsor

Scholarship at UWindsor

1991

\title{
A unified treatment of the non-relativistic and relativistic hydrogen atom: III. The reduced Green functions
}

\author{
R. A. Swainson \\ Gordon W. F. Drake \\ University of Windsor
}

Follow this and additional works at: https://scholar.uwindsor.ca/physicspub

Part of the Physics Commons

\section{Recommended Citation}

Swainson, R. A. and Drake, Gordon W. F.. (1991). A unified treatment of the non-relativistic and relativistic hydrogen atom: III. The reduced Green functions. Journal of Physics A: Mathematical and General, 24 (8), 1801-1824.

https://scholar.uwindsor.ca/physicspub/36

This Article is brought to you for free and open access by the Department of Physics at Scholarship at UWindsor. It has been accepted for inclusion in Physics Publications by an authorized administrator of Scholarship at UWindsor. For more information, please contact scholarship@uwindsor.ca. 
A unified treatment of the non-relativistic and relativistic hydrogen atom: III. The reduced Green functions

This article has been downloaded from IOPscience. Please scroll down to see the full text article.

1991 J. Phys. A: Math. Gen. 241801

(http://iopscience.iop.org/0305-4470/24/8/022)

View the table of contents for this issue, or go to the journal homepage for more

Download details:

IP Address: 137.207.184.30

The article was downloaded on 26/04/2013 at 02:43

Please note that terms and conditions apply. 


\title{
A unified treatment of the non-relativistic and relativistic hydrogen atom: III. The reduced Green functions
}

\author{
Robin A Swainson and G W F Drake \\ Department of Physics, University of Windsor, Windsor, Ontario, Canada N9B 3P4
}

Received 4 September 1990

\begin{abstract}
This is the last in a series of three papers in which it is shown how the radial part of non-relativistic and relativistic hydrogenic bound-state calculations involving the Green functions can be presented in a unified manner. The work presented here is concerned with the reduced Green functions which arise in second-order stationary state perturbation theory. Using a simple linear transformation of the four radial parts of the relativistic reduced Green function it is shown how the non-relativistic and relativistic functions are special instances of the solution of a general second-order differential equation. The general solution of this equation is exhibited in the form of a Sturmian expansion, and complete solutions in both cases are presented. Recursion relations are deduced for the radial parts of both reduced Green functions and their matrix elements are examined in detail. As a test of the given functions the second-order effect of a perturbation of the nuclear charge is calculated and is shown to agree exactly with the value expected from a simple Taylor expansion of the hydrogenic energy formula.
\end{abstract}

\section{Introduction}

This is the last in a series of three papers in which we present a unified treatment of non-relativistic and relativistic calculations involving the Coulomb Green functions (CGFs) for hydrogenic systems. In the previous two papers [1,2] we have seen how a linear transformation of the defining radial equations can be used to transform the analysis of the relativistic Dirac-Coulomb wavefunctions and Green functions (DCWFs and DCGFs) into a simple generalization of the corresponding analysis of the nonrelativistic Schrödinger-Coulomb wavefunctions and Green functions (SCWFs and SCGFs). In the present paper we extend this work to include both the non-relativistic and relativistic reduced Coulomb Green functions (RCGFs), which arise when the energy variable for the ordinary CGF coincides with an energy eigenvalue. The RCGFs are required in second-order stationary state perturbation theory as is shown in most elementary texts on quantum mechanics.

The definition of the reduced Green functions in an abstract setting is based on a consideration of the corresponding definition of the ordinary Green function. The ordinary Green function $G(z)$ corresponding to the Hamiltonian $H$ (with eigenvalues $E$ and associated eigenstates $\psi_{E}$ ) was shown in the previous paper [2] to be represented by a sum over the complete set of eigenstates of the system in the following way:

$$
G(z)=\sum \psi_{E} \psi_{E}^{\dagger} /(E-z) .
$$

It is clear from this representation that if the energy variable $z$ is equal to an eigenvalue $E^{\prime}$ of $H$ the ordinary Green function will be undefined since the denominator will be 
zero for all states corresponding to this eigenvalue. This difficulty is overcome by defining the reduced Green function $\hat{G}(z)$ to be given by

$$
\begin{aligned}
\hat{G}\left(E^{\prime}\right) & =\sum^{\prime} \psi_{E} \psi_{E}^{\dagger} /\left(E-E^{\prime}\right) \\
& =\lim _{z \rightarrow E^{\prime}}\left[G(z)-\sum \psi_{E^{\prime}} \psi_{E^{\prime}}^{\dagger} /\left(E^{\prime}-z\right)\right]
\end{aligned}
$$

where the prime on the sum in (1.2) indicates that the subspace of eigenfunctions corresponding to eigenvalue $\bar{E}^{\prime}$ is to be omitted, and the sum in (1.3) is over that subspace. An equivalent definition [3] used by several authors is

$$
\hat{G}\left(E^{\prime}\right)=\mathrm{d} /\left.\mathrm{d} z\left[\left(z-E^{\prime}\right) G(z)\right]\right|_{z=E^{\prime}} .
$$

If $H$ is represented by a differential operator $H_{r}$ acting on a Hilbert space of functions on $\mathbb{R}^{3}, \hat{G}(z)$ is itself represented by a function $\hat{G}\left(r_{1}, r_{2} ; z\right)$ on $\mathbb{R}^{3} \times \mathbb{R}^{3}$ which satisfies

$$
\left(H_{r_{1}}-E^{\prime}\right) \hat{G}\left(\boldsymbol{r}_{1}, \boldsymbol{r}_{2} ; E^{\prime}\right)=\delta\left(\boldsymbol{r}_{1}-\boldsymbol{r}_{2}\right)-\sum \psi_{E^{\prime}}\left(\boldsymbol{r}_{1}\right) \psi_{E^{\prime}}^{\dagger}\left(\boldsymbol{r}_{2}\right)
$$

which is the defining differential equation of the reduced Green functions. The RCGFs have been calculated from the known forms of the CGF using both the limiting procedure (1.3) and the differential property (1.4). In this work we will find it convenient to solve the defining differential equation (1.5) directly.

The non-relativistic reduced Schrödinger-Coulomb Green function (RsCGF) was apparently first examined in detail by Hameka [4]. Using the limiting procedure based on a calculation of the general radial SCGF he calculated the first term in the partial wave expansion of the function for the ground state, giving it in terms of products of Whittaker functions. Hostler [3] was able to find a closed form expression for the total ground state RSCGF using (1.4), and shortly thereafter derived a series expansion similar to the Sturmian expansion [5].

Two quite different forms of the radial RSCGF corresponding to a general energy level were given by Sherstyuk [6] and by Khristenko and Vetchinkin [7]. Both treatments are based on known forms for the general radial SCGF. Sherstyuk takes as his point of departure the representation of the radial SCGF as the product of Whittaker functions (see [2] equation (2.13)) and, with the aid of (1.4) derives a solution consisting of a rather complicated infinite series written in terms of $r_{>}$and $r_{<}$. This form was simplified by Johnson and Hirschfelder [8] who have presented a solution for the radial RSCGF involving finite series and known special functions, which is, nonetheless, not easy to work with and exhibits no obvious means of generalization to the relativistic problem. Khristenko and Vetchinkin [7] took as their starting point the Sturmian expansion of the radial SCGF ([2] equation (2.6)) and employed the limiting procedure embodied in (1.3) to deduce a Sturmian form for the radial RsCGF. It is this form we will reproduce here as a consequence of our unified treatment of the non-relativistic and relativistic RCGF.

Attempts to derive a compact formula for the relativistic reduced Dirac-Coulomb Green function (RDCGF) have been notably less successful. In the early seventies Zon et al [9] obtained the ground state RCGF for the second-order Dirac equation from which the ground state RDCGF could be obtained by application of a three-dimensional differential operator, though this was not explicitly done. Manakov and Zaprayagaev later [10] applied a limiting process to a form of the radial DCGF obtained using the second-order Dirac equation to find a form of the radial RDCGF for the special case of $j=n-\frac{1}{2}$. Both of these methods lead to a form of Sturmian expansion. Hylton [11], taking a quite different position found a general solution for the radial RDCGF based 
on the standard form of the radial DCGF given in terms of Whittaker functions. This solution, left in the form of various special functions and integral representations is not ideally suited to explicit calculations.

We have been able to resolve the difficulties associated with discovering general forms of the RSCGF and RDCGF by extending the methods introduced in the first two papers in this series $[1,2]$. In keeping with the general thrust of this work we have treated the angular part of the analysis in the standard manner since we believe this to be understood well enough to present few difficulties in actual calculations. The radial equations in the relativistic case have been treated as before by applying a simple linear transformation. In this way the defining radial equations for the RSCGF and the transformed RDCGF are seen to be special cases of a more general equation which we present and solve in appendix 1. That this should be so is not quite as trivial as it appeared to be for the ordinary Green functions considered in the previous paper [2]; in the present case there are functional differences between the defining radial equations for the RSCGF and transformed RDCGF. The solution obtained in appendix 1, along with a normalization condition examined in appendix 2, allows us to write down Sturmian expansions of the radial RSCGF in section 2 and of the radial RDCGF in section 3. The solution of the general relativistic RDCGF given in section 3 in a simple and useful form is, we believe, new. Other forms of the solutions, such as those presented for the ordinary radial CGFs in [2] are not available for the RCGFs. In section 4 we derive various recursion relations between different radial RCGFs which have been given only for the non-relativistic functions in previous studies but which we present for the relativistic functions too. These recursion relations are obtained simultaneously for both the non-relativistic and the relativistic functions using the results presented in appendix 3 . In section 5 we show how one might go about calculating matrix elements of the Green functions. Again the difference in the method for the two cases is minor; we have even been able to give a form of the hypervirial theorem applicable in both cases.

Very often calculations which seem to involve the reduced CGF do so in name only; the functions involved are actually general CGFs evaluated at an energy eigenvalue. Such calculations include the polarizabilities of the hydrogen atom. We thought it important to test our solutions for the RDCGF directly, and this we do in section 6 where we calculate the second-order energy shift due to a small perturbation of the nuclear charge. Obviously this quantity can be found from the Dirac formula for the hydrogenic energy levels; our calculation consists then of a sharp test of the main results of this paper. Section 6 also contains some concluding remarks to this series of papers.

\section{The reduced Schrödinger-Coulomb Green function}

The reduced Schrödinger-Coulomb Green function, $\hat{G}\left(r_{1}, r_{2} ; n\right)$ corresponding to the energy $E_{n}$ is defined to be the solution, subject to appropriate boundary conditions, of

$\left(-\frac{\hbar^{2}}{2 m} \nabla_{1}^{2}-\frac{\hbar^{2}}{a m r_{1}}-E_{n}\right) \hat{G}\left(\boldsymbol{r}_{1}, \boldsymbol{r}_{2} ; n\right)=\delta\left(\boldsymbol{r}_{1}-\boldsymbol{r}_{2}\right)-\sum \psi_{n l m}\left(\boldsymbol{r}_{1}\right) \psi_{n I m}\left(\boldsymbol{r}_{2}\right)^{*}$

where $\psi_{n l m}(\boldsymbol{r})=R_{n l}(r) Y_{l m}(\hat{\boldsymbol{r}})$ is the Schrödinger-Coulomb wavefunction defined in [1], and the sum is over the entire manifold of states with energy $E_{n}$. A functional form of $\hat{G}$ suitable for use in specific calculations can be found by following basically 
the same analysis as was used in solving the ordinary Schrödinger-Coulomb Green function [2]. We begin by expanding $\hat{G}$ in terms of spherical harmonics [12],

$$
\hat{G}\left(\boldsymbol{r}_{1}, \boldsymbol{r}_{2} ; n\right)=\sum_{l m} \hat{g}_{l}\left(r_{1}, r_{2} ; n\right) Y_{l m}\left(\hat{r}_{1}\right) Y_{l m}^{*}\left(\hat{r}_{2}\right)
$$

and substitute this expansion into (2.1). Application of the orthogonality conditions for spherical harmonics leads to two radial equations for $\hat{g}$ :

$$
\begin{aligned}
\left(\frac{\mathrm{d}^{2}}{\mathrm{~d} r_{1}^{2}}+\frac{2}{r_{1}} \frac{\mathrm{d}}{\mathrm{d} r_{1}}\right. & \left.-\frac{l(l+1)}{r_{1}^{2}}+\frac{2}{a r_{1}}-\frac{1}{a^{2} n^{2}}\right) \hat{g}_{l}\left(r_{1}, r_{2} ; n\right) \\
& =-\left(2 m / \hbar^{2}\right) \frac{\delta\left(r_{1}-r_{2}\right)}{r_{1} r_{2}} \quad \text { for } l \geqslant n \\
& =-\left(2 m / \hbar^{2}\right)\left(\frac{\delta\left(r_{1}-r_{2}\right)}{r_{1} r_{2}}-R_{n l}\left(r_{1}\right) R_{n l}\left(r_{2}\right)\right) \quad \text { for } l<n .
\end{aligned}
$$

It is immediately clear that for $l \geqslant n$ the reduced radial functions are identical to the full radial functions obtained in [2],

$$
\hat{g}_{l}\left(r_{1}, r_{2} ; n\right)=g_{l}\left(r_{1}, r_{2} ; n\right) \quad \text { for } l \geqslant n
$$

with the energy variable fixed at the eigenvalue. The solution of the second equation requires first a discussion of the appropriate boundary conditions.

Consideration of the forms of the full radial Green function and the radial wavefunctions leads us to require that

$$
\begin{aligned}
& \hat{g}_{l}\left(r_{1}, r_{2} ; n\right) \rightarrow \mathrm{O}\left(r_{1}^{l}\right) \quad \text { as } \quad r_{1} \rightarrow 0 \\
& \hat{g}_{l}\left(r_{1}, r_{2} ; n\right) \rightarrow \mathrm{e}^{-r_{1} / a n} \quad \text { as } \quad r_{1} \rightarrow \infty
\end{aligned}
$$

and

$$
\hat{g}_{l}\left(r_{1}, r_{2} ; n\right)=\hat{g}_{l}\left(r_{2}, r_{1} ; n\right) .
$$

We further require that $\hat{g}_{1}$ be unique. It follows from the general definition of the reduced Green functions that they must be orthogonal to the relevant energy eigenspace. Since adding terms in $R_{n l}$ to $\hat{g}_{l}$ will not affect $(2.3 b)$ we make the following additional restriction on $\hat{g}_{1}$ :

$$
\int_{0}^{\infty} R_{n !}\left(r_{1}\right) \hat{g}_{1}\left(r_{1}, r_{2} ; n\right) r_{1}^{2} \mathrm{~d} r_{1}=0
$$

and similarly for $r_{2}$. With these formalities over, we can now quite rapidly solve equation $(2.3 b)$. In fact a somewhat more general equation is solved in appendix 1 , and the solution to $(2.3 b)$ follows from the solution presented there with $\nu=n, \lambda=l$, and $\omega=1 /$ an. Thus, in the notation of appendix 1

$$
\hat{g}_{l}\left(r_{1}, r_{2} ; n\right)=+2 m / \hbar^{2} \hat{g}_{n l}\left(r_{1}, r_{2} ; 1 / a n\right) .
$$

The values of $\sigma_{\lambda}$ and $\tau_{\lambda}$ are obtained from the definition of $R_{n l}$ given in [1]:

$$
\sigma_{l}=(2 / a n)^{2 l}\left(4 / n^{4} a^{3}\right)(n-l-1) ! /(n+l) ! \quad \tau_{!}=0 .
$$


The arbitrary constant $A_{l}$ is fixed by the normalization condition (2.6), which can be readily calculated given the analysis presented in appendix 2 . In fact, in this case $A_{l}=1$. Thus the final form of the solution is

$$
\begin{aligned}
\hat{g}_{l}\left(r_{1}, r_{2} ; n\right)= & +2 m / \hbar^{2}(2 / a n)^{2 l+1}\left(r_{1} r_{2}\right)^{l} \mathrm{e}^{-\left(r_{1}+r_{2}\right) / a n} \\
& \times\left(\sum_{i=0}^{\infty} \frac{i !}{(2 l+1+i) !(l+1+i-n)} L_{i}^{2 l+1}\left(2 r_{1} / a n\right) L_{i}^{2 l+1}\left(2 r_{2} / a n\right)\right. \\
& +\frac{(n-l-1) !}{2 n(n+l) !}\left\{L _ { n - l - 1 } ^ { 2 l + 1 } ( 2 r _ { 1 } / a n ) \left[(n-l) L_{n-l}^{2 l+1}\left(2 r_{2} / a n\right)\right.\right. \\
& \left.-(n+l) L_{n-l-2}^{2 l+1}\left(2 r_{2} / a n\right)\right]+L_{n-l-1}^{2 l+1}\left(2 r_{1} / a n\right) L_{n-l-1}^{2 l+1}\left(2 r_{2} / a n\right) \\
& \left.\left.+\left[(n-l) L_{n-l}^{2 l+1}\left(2 r_{1} / a n\right)-(n+l) L_{n-l-2}^{2 l+1}\left(2 r_{1} / a n\right)\right] L_{n-l-1}^{2 l+1}\left(2 r_{2} / a n\right)\right\}\right)
\end{aligned}
$$

The asterisk appearing on the summation sign indicates that the term for $i=n-l-1$ is to be omitted.

In the previous paper [2] various properties of special functions were employed to show how many other functional forms of the radial scGF could be derived from the Sturmian form. Similar methods cannot be employed here because of the (omitted) singularity within the infinite sum. Nonetheless the Sturmian form for the radial RSCGF given in (2.9) can be shown to be appropriate for many applications. This will be, therefore, the only form we present in this paper.

A solution of (2.1) corresponding to the form of the radial sCGF given in terms of Whittaker functions [2] has been given by Johnson and Hirschfelder [8]. However, certain terms in that solution corresponding to products of Laguerre polynomials have been obscured by the complicated analysis involved, and the possibility of extending the presentation as a whole to the relativistic case is not obvious. An integral solution, bypassing the separation into angular and radial parts has been obtained by Hostler [3]; again the relevance to the relativistic case is unclear.

\section{The reduced Dirac-Coulomb Green function}

As in the previous section, our treatment here of the RDCGF follows closely the development of the full DCGF we presented in [2], and indeed we retain the notation of that paper. The Dirac-Coulomb wavefunctions given in [1] will also be employed extensively in the following. At the outset, however, we note that the energy eigenvalues $\varepsilon_{N_{\gamma}}$ depend on $|\kappa|$ alone; thus, whereas in the non-relativistic case there are $n-1$ values of $l$ with energy corresponding to the principal quantum number $n$, in the relativistic case only two values of $\kappa, \pm|\kappa|$, have the same energy for given $N$. Thus, in some ways the RDCGF is easier to deal with than the RSCGF.

The RDCGF $\hat{G}\left(r_{1}, r_{2} ; N, \gamma\right)$ corresponding to the energy eigenvalue $\varepsilon_{N \gamma}$ is the $4 \times 4$ matrix function satisfying

$$
\begin{aligned}
& {\left[\left(\boldsymbol{\alpha} \cdot \hat{r}_{1}\right)\left(-\frac{\mathrm{i} \hbar c}{r_{1}} \frac{d}{d r_{1}} r_{1}+\frac{\mathrm{i} c}{r_{1}} \beta K\right)+\beta m c^{2}-\frac{\alpha Z \hbar c}{r_{1}}-\omega_{N \gamma}\right] \hat{G}\left(\boldsymbol{r}_{1}, \boldsymbol{r}_{2} ; N, \gamma\right)} \\
& =\delta\left(r_{1}-r_{2}\right) I_{4}-\sum\left[\Psi_{N \kappa M}\left(r_{1}\right) \Psi_{N \kappa M}\left(r_{2}\right)^{\dagger}+\Psi_{N-\kappa M}\left(r_{1}\right) \Psi_{N-\kappa M}\left(r_{2}\right)^{\dagger}\right]
\end{aligned}
$$


subject to suitable boundary conditions, and where the summation is for $M=|\kappa| \pm \frac{1}{2}$. Following the analysis for the general DCGF [2] we expand $\hat{G}$ and $\Psi$ in terms of the spinor spherical harmonics [12],

$$
\chi_{\kappa}^{M}(\theta, \varphi)=\left(\begin{array}{c}
\frac{-\kappa}{|\kappa|}\left(\frac{\kappa+1 / 2-M}{2 \kappa+1}\right)^{1 / 2} Y_{|\kappa+1 / 2|-1 / 2, M-1 / 2}(\theta, \varphi) \\
\left(\frac{\kappa+1 / 2+M}{2 \kappa+1}\right)^{1 / 2} Y_{|\kappa+1 / 2|-1 / 2, M+1 / 2}(\theta, \varphi)
\end{array}\right)
$$

that is we let

$\hat{G}\left(\boldsymbol{r}_{1}, \boldsymbol{r}_{2} ; N, \gamma\right)=\sum_{\mathrm{K} \mu}\left(\begin{array}{cc}\hat{\boldsymbol{g}}_{k}^{11}\left(r_{1}, r_{2}\right) \chi_{k}^{\mu}\left(\hat{\boldsymbol{r}}_{1}\right) \chi_{k}^{\mu \dagger}\left(\hat{\boldsymbol{r}}_{2}\right) & -\mathrm{i} \hat{g}_{k}^{12}\left(r_{1}, r_{2}\right) \chi_{k}^{\mu}\left(\hat{\boldsymbol{r}}_{1}\right) \chi_{-k}^{\mu \dagger}\left(\hat{\boldsymbol{r}}_{2}\right) \\ \mathrm{i} \hat{g}_{k}^{21}\left(r_{1}, r_{2}\right) \chi_{-k}^{\mu}\left(\hat{\boldsymbol{r}}_{1}\right) \chi_{k}^{\mu \dagger}\left(\hat{\boldsymbol{r}}_{2}\right) & \hat{\boldsymbol{g}}_{k}^{22}\left(r_{1}, r_{2}\right) \chi_{-k}^{\mu}\left(\hat{\boldsymbol{r}}_{1}\right) \chi_{-k}^{\mu \dagger}\left(\hat{\boldsymbol{r}}_{2}\right)\end{array}\right)$

and

$$
\Psi_{N \kappa M}(\boldsymbol{r})=\left(\begin{array}{c}
f_{1}^{N \kappa}(r) \chi_{\kappa}^{M}(\hat{\boldsymbol{r}}) \\
\mathrm{i} f_{2}^{N \kappa}(r) \chi_{-\kappa}^{M}(\hat{\boldsymbol{r}})
\end{array}\right) .
$$

Thus, as in the case of the radial RSCGF two separate situations obtain:

$$
\begin{aligned}
\left(\begin{array}{cc}
\left(\varepsilon_{0}-\varepsilon_{N \gamma}-\alpha Z / r_{1}\right) & \left(-\frac{1}{r_{1}} \frac{\mathrm{d}}{\mathrm{d} r_{1}} r_{1}+\frac{k}{r_{1}}\right) \\
\left(\frac{1}{r_{1}} \frac{\mathrm{d}}{\mathrm{d} r_{1}} r_{1}+\frac{k}{r_{1}}\right) & \left(-\varepsilon_{0}-\varepsilon_{N \gamma}-a Z / r_{1}\right)
\end{array}\right)\left(\begin{array}{ll}
\hat{g}_{k}^{11} & \hat{g}_{k}^{12} \\
\hat{g}_{k}^{21} & \hat{g}_{k}^{22}
\end{array}\right) \\
=\frac{\delta\left(r_{1}-r_{2}\right)}{r_{1} r_{2}} I_{2} / \hbar c \quad k \neq \pm \kappa \\
=\frac{\delta\left(r_{1}-r_{2}\right)}{\check{r}_{1} r_{2}} I_{2} / \hbar c-\left(\begin{array}{l}
f_{1}^{N k}\left(r_{1}\right) \\
f_{2}^{N k}\left(r_{1}\right)
\end{array}\right)\left(f_{1}^{N k}\left(r_{2}\right) \quad f_{2}^{N k}\left(r_{2}\right)\right) \quad k= \pm \kappa .
\end{aligned}
$$

For $k \neq \pm \kappa$ the radial RDCGF is thus precisely the same as the general radial DCGF given in the previous paper, evaluated at $\varepsilon=\varepsilon_{N \gamma}$. Thus the analysis presented in [2] is immediately applicable and the problem is solved.

In the case where $k= \pm \kappa$ we have a somewhat more difficult equation to deal with. Nonetheless a solution along the lines established in [1] and [2] is possible, and this we now present. As before we let

$$
\left(\begin{array}{l}
g_{1}^{N k}(r) \\
g_{2}^{N k}(r)
\end{array}\right)=\underline{X}_{k}\left(\begin{array}{l}
f_{1}^{N k}(r) \\
f_{2}^{N k}(r)
\end{array}\right)
$$

and

$$
\left(\begin{array}{ll}
\hat{h}_{k}^{11}\left(r_{1}, r_{2}\right) & \hat{h}_{k}^{12}\left(r_{1}, r_{2}\right) \\
\hat{h}_{k}^{21}\left(r_{1}, r_{2}\right) & \hat{h}_{k}^{22}\left(r_{1}, r_{2}\right)
\end{array}\right)=\underline{X}_{k}\left(\begin{array}{cc}
\hat{g}_{k}^{11}\left(r_{1}, r_{2}\right) & \hat{g}_{k}^{12}\left(r_{1}, r_{2}\right) \\
\hat{g}_{k}^{21}\left(r_{1}, r_{2}\right) & \hat{g}_{k}^{22}\left(r_{1}, r_{2}\right)
\end{array}\right) \underline{X}_{k}
$$

where

$$
\underline{X}_{k}=\left(\begin{array}{cc}
1 & X_{k} \\
X_{k} & 1
\end{array}\right) \quad X_{k}=(-k+\gamma) / \alpha Z \quad \gamma=\sqrt{k^{2}-\alpha^{2} Z^{2}}
$$


Then

$$
\begin{aligned}
& \left(\begin{array}{cc}
\left(\varepsilon_{0}-\varepsilon_{N \gamma} k / \gamma\right) & \left(\frac{-\alpha Z}{\gamma} \varepsilon_{N \gamma}+\frac{\gamma-1}{r_{1}}-\frac{\mathrm{d}}{\mathrm{d} r_{1}}\right) \\
\left(\frac{-\alpha Z}{\gamma} \varepsilon_{N_{\gamma}}+\frac{\gamma+1}{r_{1}}+\frac{\mathrm{d}}{\mathrm{d} r_{1}}\right) & \left(-\varepsilon_{0}-\varepsilon_{N \gamma} k / \gamma\right)
\end{array}\right)\left(\begin{array}{ll}
\hat{h}_{k}^{11}\left(r_{1}, r_{2}\right) & \hat{h}_{k}^{12}\left(r_{1}, r_{2}\right) \\
\hat{h}_{k}^{21}\left(r_{1}, r_{2}\right) & \hat{h}_{k}^{22}\left(r_{1}, r_{2}\right)
\end{array}\right) \\
& =\left(1-X_{k}^{2}\right) / \hbar c \frac{\delta\left(r_{1}-r_{2}\right)}{r_{1} r_{2}} I_{2}-1 / \gamma\left(\begin{array}{cc}
k & \alpha Z \\
\alpha Z & k
\end{array}\right)\left(\begin{array}{l}
g_{1}^{N k}\left(r_{1}\right) \\
g_{2}^{N k}\left(r_{1}\right)
\end{array}\right)\left(g_{1}^{N k}\left(r_{2}\right) g_{2}^{N k}\left(r_{2}\right)\right) \text {. }
\end{aligned}
$$

The set of four equations embodied in (3.10) separate into two pairs which then simplify further. The first pair, relating $\hat{h}_{k}^{11}$ and $\hat{h}_{k}^{21}$, is

$$
\begin{aligned}
\left(\gamma \varepsilon_{0}-\varepsilon_{N \gamma} k\right) \hat{h}_{k}^{11}\left(r_{1}, r_{2}\right)+ & {\left[-\alpha Z \varepsilon_{N \gamma}+\gamma\left(\frac{\gamma-1}{r_{1}}-\frac{\mathrm{d}}{\mathrm{d} r_{1}}\right)\right] \hat{h}_{k}^{21}\left(r_{1}, r_{2}\right) } \\
= & \gamma\left(1-X_{k}^{2}\right) \frac{\delta\left(r_{1}-r_{2}\right)}{\ddot{r}_{1} \ddot{r}_{2}} / \hbar c-\left[k g_{1}^{N k}\left(r_{1}\right)\right. \\
& \left.+\alpha Z g_{2}^{N k}\left(r_{1}\right)\right] g_{1}^{N k}\left(r_{2}\right) / \hbar c \\
{\left[-\alpha Z \varepsilon_{N \gamma}+\right.} & \left.\gamma\left(\frac{\gamma+1}{r_{1}}+\frac{\mathrm{d}}{\mathrm{d} r_{1}}\right)\right] \hat{h}_{k}^{11}\left(r_{1}, r_{2}\right)-\left(\gamma \varepsilon_{0}+\varepsilon_{N \gamma} k\right) \hat{h}_{k}^{21}\left(r_{1}, r_{2}\right) \\
= & -\left[\alpha Z g_{1}^{N k}\left(r_{1}\right)+k g_{2}^{N k}\left(r_{1}\right)\right] g_{1}^{N k}\left(r_{2}\right) / \hbar c .
\end{aligned}
$$

We now substitute (3.11b) into (3.11a) to eliminate the off-diagonal term; we have the added problem of dealing with the wavefunctions however. The analysis is simplified using the recursion relations for the relativistic wavefunctions derived in the first paper of this series, and which we reproduce here for clarity:

$$
\begin{aligned}
& {\left[\gamma\left(\frac{\mathrm{d}}{\mathrm{d} r}+\frac{\gamma+1}{r}\right)-\alpha Z \varepsilon_{N \gamma}\right] g_{1}^{N k}(r)=\left(\gamma \varepsilon_{0}+\varepsilon_{N \gamma} k\right) g_{2}^{N k}(r)} \\
& {\left[\gamma\left(\frac{\mathrm{d}}{\mathrm{d} r}-\frac{\gamma-1}{r}\right)+\alpha Z \varepsilon_{N_{\gamma}}\right] g_{2}^{N k}(r)=\left(\gamma \varepsilon_{0}-\varepsilon_{N \gamma} k\right) g_{1}^{N k}(r) .}
\end{aligned}
$$

Then (3.11a) and (3.11b) imply that

$$
\begin{aligned}
\hbar c\left(\frac{\mathrm{d}^{2}}{\mathrm{~d} r_{1}^{2}}+\frac{2}{r_{1}}\right. & \left.\frac{\mathrm{d}}{\mathrm{d} r_{1}}-\frac{\gamma(\gamma+1)}{r_{1}^{2}}+\frac{2 \alpha Z}{r_{1}} \varepsilon_{N \gamma}-\omega_{N \gamma}^{2}\right) \hat{h}_{k}^{11}\left(r_{1}, r_{2}\right) \\
& =\left(\varepsilon_{0}+\varepsilon_{N \gamma} k / \gamma\right)\left(1-X_{k}^{2}\right) \frac{\delta\left(r_{1}-r_{2}\right)}{r_{1} r_{2}}+2\left(\varepsilon_{N \gamma}+\alpha Z / r_{1}\right) g_{1}^{N k}\left(r_{1}\right) g_{1}^{N k}\left(r_{2}\right)
\end{aligned}
$$

and

$$
\begin{gathered}
\hat{h}_{k}^{21}\left(r_{1}, r_{2}\right)=1 /\left(\varepsilon_{N \gamma} k+\varepsilon_{0} \gamma\right)\left[\gamma\left(\frac{\mathrm{d}}{\mathrm{d} r_{1}}+\frac{\gamma+1}{r_{1}}\right)-\alpha Z \varepsilon_{N \gamma}\right] \hat{h}_{k}^{11}\left(r_{1}, r_{2}\right) \\
+\left[\alpha Z g_{1}^{N k}\left(r_{1}\right)+k g_{2}^{N k}\left(r_{1}\right)\right] g_{1}^{N k}\left(r_{2}\right) / \hbar c .
\end{gathered}
$$


The second pair of equations is

$$
\begin{aligned}
& \left(\varepsilon_{0}-\varepsilon_{N \gamma} k / \gamma\right) \hat{h}_{k}^{12}\left(r_{1}, r_{2}\right)+\left(-\frac{\alpha Z}{\gamma} \varepsilon_{N \gamma}+\frac{\gamma-1}{r_{1}}-\frac{d}{d r_{1}}\right) \hat{h}_{k}^{22}\left(r_{1}, r_{2}\right) \\
& =-\left(k g_{1}^{N k}\left(r_{1}\right)+\alpha Z g_{2}^{N k}\left(r_{1}\right)\right] g_{2}^{N k}\left(r_{2}\right) / \hbar c \\
& \left(-\frac{\alpha Z}{\gamma} \varepsilon_{N_{\gamma}}+\frac{\gamma+1}{r_{1}}+\frac{\mathrm{d}}{\mathrm{d} r_{1}}\right) \hat{h}_{k}^{12}\left(r_{1}, r_{2}\right)-\left(\varepsilon_{0}+\varepsilon_{N \gamma} k / \gamma\right) \hat{h}_{k}^{22}\left(r_{1}, r_{2}\right) \\
& =\gamma\left(1-X_{k}^{2}\right) / \hbar c \frac{\delta\left(r_{1}-r_{2}\right)}{r_{1} r_{2}}-\left[\alpha Z g_{1}^{N k}\left(r_{1}\right)+k g_{2}^{N k}\left(r_{1}\right)\right] g_{2}^{N k}\left(r_{2}\right) / \hbar c .
\end{aligned}
$$

On substituting (3.14a) into (3.14b) and using (3.12a) and (3.12b) we find,

$$
\begin{aligned}
\hbar c\left(\frac{\mathrm{d}^{2}}{\mathrm{~d} r_{1}^{2}}+\frac{2}{r_{1}}\right. & \left.\frac{\mathrm{d}}{\mathrm{d} r_{1}}-\frac{\gamma(\gamma-1)}{r_{1}^{2}}+\frac{2 \alpha Z}{r_{1}} \varepsilon_{N \gamma}-\omega_{N \gamma}^{2}\right) \hat{h}_{k}^{22}\left(r_{1}, r_{2}\right) \\
& =\frac{\left(\gamma \varepsilon_{0}-\varepsilon_{N \gamma} k\right)\left(1-X_{k}^{2}\right)}{\gamma} \frac{\delta\left(r_{1}-r_{2}\right)}{r_{1} r_{2}}+2\left(\varepsilon_{N \gamma}+\alpha Z / r_{1}\right) g_{2}^{N k}\left(r_{1}\right) g_{2}^{N k}\left(r_{2}\right)
\end{aligned}
$$

and

$$
\begin{aligned}
\hat{h}_{k}^{12}\left(r_{1}, r_{2}\right)=- & \gamma /\left(\varepsilon_{N \gamma} k-\varepsilon_{0} \gamma\right)\left[\gamma\left(\frac{\mathrm{d}}{\mathrm{d} r_{1}}-\frac{\gamma-1}{r_{1}}\right)+\alpha Z \varepsilon_{N \gamma}\right] \hat{h}_{k}^{22}\left(r_{1}, r_{2}\right) \\
- & {\left[k g_{1}^{N k}\left(r_{1}\right)+\alpha Z g_{2}^{N k}\left(r_{1}\right)\right] g_{2}^{N k}\left(r_{2}\right) / \hbar c . }
\end{aligned}
$$

The form of both ( $3.13 a)$ and (3.15a) is remarkably simple; these two equations, along with the radial equation for the RSCGF, are special cases of a more general equation, the solution of which is derived in appendix 1 . The appropriate boundary conditions can be readily inferred from the solution of the ordinary DCGF. Referring to (A1.9), and since in this case, in the notation of appendix 1 ,

$$
\begin{aligned}
& \sigma_{\gamma}=\left(2 \omega_{N \gamma}\right)^{2 \gamma+3}\left(\varepsilon_{N \gamma}^{2} / 2 \varepsilon_{0}^{2}\right)(N-1) ! /[\Gamma(2 \gamma+N+1)(N+\gamma)] \quad \tau_{\gamma}=\alpha Z \sigma_{\gamma} / \varepsilon_{N \gamma} \\
& \sigma_{\gamma-1}=\left(2 \omega_{N \gamma}\right)^{2 \gamma+1}\left(\varepsilon_{N \gamma}^{2} / 2 \varepsilon_{0}^{2}\right) N ! /[\Gamma(2 \gamma+N)(N+\gamma)] \quad \tau_{\gamma-1}=\alpha Z \sigma_{\gamma} / \varepsilon_{N \gamma}
\end{aligned}
$$

we can immediately write down expressions for the diagonal radial RDCGF. However, it is advantageous to consider the orthogonality condition we are imposing on $\hat{G}$,

$$
\int \Psi_{N k}^{\dagger}\left(\boldsymbol{r}_{1}\right) \hat{G}\left(\boldsymbol{r}_{1}, \boldsymbol{r}_{2} ; N, \gamma\right) \mathrm{d} \boldsymbol{r}_{1}=0 \quad k= \pm \kappa .
$$

Expanding $\Psi$ and $\hat{G}$ in spinor spherical harmonics, and transforming the radial RDCGFs according to (3.8) leads us to two independent orthogonality conditions:

$$
\begin{aligned}
& \int_{0}^{\infty}\left\{\left[\left(k g_{1}^{N k}\left(r_{1}\right)+\alpha Z g_{2}^{N k}\left(r_{1}\right)\right] \hat{h}_{k}^{11}\left(r_{1}, r_{2}\right)\right.\right. \\
& \left.+\left[\alpha Z g_{1}^{N k}\left(r_{1}\right)+k g_{2}^{N k}\left(r_{1}\right)\right] \hat{h}_{k}^{21}\left(r_{1}, r_{2}\right)\right\} r_{1}^{2} \mathrm{~d} r_{1}=0 \\
& \int_{0}^{\infty}\left\{\left[\left(k g_{1}^{N k}\left(r_{1}\right)+\alpha Z g_{2}^{N k}\left(r_{1}\right)\right] \hat{h}_{k}^{12}\left(r_{1}, r_{2}\right)\right.\right. \\
& \left.+\left[\alpha Z g_{1}^{N k}\left(r_{1}\right)+k g_{2}^{N k}\left(r_{1}\right)\right] \hat{h}_{k}^{22}\left(r_{1}, r_{2}\right)\right\} r_{1}^{2} \mathrm{~d} r_{1}=0 .
\end{aligned}
$$


Expressing the integrands in terms of the diagonal elements only, performing partial integrations where necessary and using the recursion relations for the radial wavefunctions, leads to the following two equivalent conditions:

$$
\begin{aligned}
2 \gamma^{2} \int_{0}^{\infty}\left(\varepsilon_{N y}\right. & \left.+\alpha Z / r_{1}\right) g_{1}^{N k}\left(r_{1}\right) \hat{h}_{k}^{11}\left(r_{1}, r_{2}\right) r_{1}^{2} \mathrm{~d} r_{1} \\
& +\int_{0}^{\infty}\left[\alpha Z g_{1}^{N k}\left(r_{1}\right)+k g_{2}^{N k}\left(r_{1}\right)\right]^{2} r_{1}^{2} \mathrm{~d} r_{1} g_{1}^{N k}\left(r_{2}\right) / \hbar c=0
\end{aligned}
$$

$2 \gamma^{2} \int_{0}^{\infty}\left(\varepsilon_{N \gamma}+\alpha Z / r_{1}\right) g_{2}^{N k}\left(r_{1}\right) \hat{h}_{k}^{22}\left(r_{1}, r_{2}\right) r_{1}^{2} \mathrm{~d} r_{1}$

$$
+\int_{0}^{\infty}\left[k g_{1}^{N k}\left(r_{1}\right)+\alpha Z g_{2}^{N k}\left(r_{1}\right)\right]^{2} r_{1}^{2} \mathrm{~d} r_{1} g_{2}^{N k}\left(r_{2}\right) / \hbar c=0 .
$$

The integrals involving the Green functions are special cases of one performed in appendix 2, while the integrals of the wavefunctions can be performed using the results of [1] (appendix 3). We find then that

$A_{\gamma}=1-2 k \omega_{N \gamma}^{2} /\left[\varepsilon_{N \gamma}\left(\varepsilon_{N \gamma} k+\varepsilon_{0} \gamma\right)\right] \quad A_{\gamma-1} \equiv 1-2 k \omega_{N \gamma}^{2} /\left[\varepsilon_{N \gamma}\left(\varepsilon_{N \gamma} k-\varepsilon_{0} \gamma\right)\right]$.

We are now in a position to write down the diagonal elements of the radial RDCGF, which are

$$
\begin{aligned}
\hat{h}_{k}^{11}\left(r_{1}, r_{2}\right)=2 & \omega_{N \gamma}\left(\varepsilon_{N \gamma} k+\varepsilon_{0} \gamma\right) /[\hbar c(k+\gamma)]\left(2 \omega_{N \gamma} r_{1}\right)^{\gamma}\left(2 \omega_{N \gamma} r_{2}\right)^{\gamma} \mathrm{e}^{-\left(r_{1}+r_{2}\right) \omega_{N \gamma}} \\
& \times\left(2 \sum_{i=0}^{\infty} \frac{i !}{\Gamma(2 \gamma+2+i)(i+1-N)} L_{i}^{2 \gamma+1}\left(2 \omega_{N \gamma} r_{1}\right) L_{i}^{2 \gamma+1}\left(2 \omega_{N \gamma} r_{2}\right)\right. \\
& +\left(\varepsilon_{N \gamma}^{2} / \varepsilon_{0}^{2}\right) \frac{(N-1) !}{\Gamma(2 \gamma+N+1)(N+\gamma)} \\
& \times\left\{\left[N L_{N}^{2 \gamma+1}\left(2 \omega_{N \gamma} r_{1}\right)-(2 \gamma+N) L_{N-2}^{2 \gamma+1}\left(2 \omega_{N \gamma} r_{1}\right)\right] L_{N-1}^{2 \gamma+1}\left(2 \omega_{N \gamma} r_{2}\right)\right. \\
& +\left(1-2 k \omega_{N \gamma}^{2} /\left[\varepsilon_{N \gamma}\left(\varepsilon_{N \gamma} k+\varepsilon_{0} \gamma\right)\right]\right) L_{N-1}^{2 \gamma+1}\left(2 \omega_{N \gamma} r_{1}\right) L_{N-1}^{2 \gamma+1}\left(2 \omega_{N \gamma} r_{2}\right) \\
& \left.\left.+L_{N-1}^{2 \gamma+1}\left(2 \omega_{N \gamma} r_{1}\right)\left[N L_{N}^{2 \gamma+1}\left(2 \omega_{N \gamma} r_{2}\right)-(2 \gamma+N) L_{N-2}^{2 \gamma+1}\left(2 \omega_{N \gamma} r_{2}\right)\right]\right\}\right)
\end{aligned}
$$

and

$$
\begin{aligned}
\hat{h}_{k}^{22}\left(r_{1}, r_{2}\right)=2 & \omega_{N \gamma}\left(\varepsilon_{N \gamma} k-\varepsilon_{0} \gamma\right) /[\hbar c(k+\gamma)]\left(2 \omega_{N \gamma} r_{1}\right)^{\gamma-1}\left(2 \omega_{N \gamma} r_{2}\right)^{\gamma-1} \mathrm{e}^{-\left(r_{1}+r_{2}\right) \omega_{N \gamma}} \\
& \times\left(2 \sum_{i=0}^{\infty} \frac{i !}{\Gamma(2 \gamma+i)(i-N)} L_{i}^{2 \gamma-1}\left(2 \omega_{N \gamma} r_{1}\right) L_{i}^{2 \gamma-1}\left(2 \omega_{N \gamma} r_{2}\right)\right. \\
& +\left(\varepsilon_{N \gamma}^{2} / \varepsilon_{0}^{2}\right) \frac{N !}{\Gamma(2 \gamma+N)(N+\gamma)} \\
& \times\left\{\left[(N+1) L_{N+1}^{2 \gamma-1}\left(2 \omega_{N \gamma} r_{1}\right)-(2 \gamma+N-1) L_{N-1}^{2 \gamma-1}\left(2 \omega_{N \gamma} r_{1}\right)\right] L_{N}^{2 \gamma-1}\left(2 \omega_{N \gamma} r_{2}\right)\right. \\
& +\left(1-2 k \omega_{N \gamma}^{2} /\left[\varepsilon_{N \gamma}\left(\varepsilon_{N \gamma} k-\varepsilon_{0} \gamma\right)\right]\right) L_{N}^{2 \gamma-1}\left(2 \omega_{N \gamma} r_{1}\right) L_{N}^{2 \gamma-1}\left(2 \omega_{N \gamma} r_{2}\right) \\
& \left.\left.+L_{N}^{2 \gamma-1}\left(2 \omega_{N \gamma} r_{1}\right)\left[(N+1) L_{N+1}^{2 \gamma-1}\left(2 \omega_{N \gamma} r_{2}\right)-(2 \gamma+N-1) L_{N-1}^{2 \gamma-1}\left(2 \omega_{N \gamma} r_{2}\right)\right]\right\}\right)
\end{aligned}
$$


Notice that $\hat{h}^{11}$ and $\hat{h}^{22}$ are symmetrical in $r_{1}$ and $r_{2}$. This is not the case for the off-diagonal terms, which can be easily deduced using the results of appendix 3 . Thus $(3.11 b)$ and (A3.8), together with the explicit forms of the Dirac-Coulomb wavefunction given in [1], imply that

$$
\begin{aligned}
\hat{h}_{k}^{21}\left(r_{1}, r_{2}\right)=1 /[ & \hbar c(k+\gamma)] \delta\left(r_{1}-r_{2}\right) / \sqrt{r_{1} r_{2}} \\
& +2 \omega_{N \gamma}^{2} /[\hbar c(k+\gamma)]\left(2 \omega_{N \gamma} r_{1}\right)^{\gamma-1}\left(2 \omega_{N \gamma} r_{2}\right)^{\gamma} \mathrm{e}^{-\left(r_{1}+r_{2}\right) \omega_{N \gamma}} \\
& \times\left(2 \sum_{i=0}^{\infty} \frac{(i+1) !}{\Gamma(2 \gamma+1+i)(i+1-N)} L_{i+1}^{2 \gamma-1}\left(2 \omega_{N \gamma} r_{1}\right) L_{i}^{2 \gamma+1}\left(2 \omega_{N \gamma} r_{2}\right)\right. \\
& +\left(\varepsilon_{N \gamma}^{2} / \varepsilon_{0}^{2}\right) \frac{N !}{\Gamma(2 \gamma+N)(N+\gamma)} \\
& \times\left\{\left[(N+1) L_{N+1}^{2 \gamma-1}\left(2 \omega_{N \gamma} r_{1}\right)-(2 \gamma+N-1) L_{N-1}^{2 \gamma-1}\left(2 \omega_{N \gamma} r_{1}\right)\right] L_{N-1}^{2 \gamma+1}\left(2 \omega_{N \gamma} r_{2}\right)\right. \\
& +3 L_{N}^{2 \gamma-1}\left(2 \omega_{N \gamma} r_{1}\right) L_{N-1}^{2 \gamma+1}\left(2 \omega_{N \gamma} r_{2}\right) \\
& \left.\left.+L_{N}^{2 \gamma-1}\left(2 \omega_{N \gamma} r_{1}\right)\left[N L_{N}^{2 \gamma+1}\left(2 \omega_{N \gamma} r_{2}\right)-(2 \gamma+N) L_{N-2}^{2 \gamma+1}\left(2 \omega_{N \gamma} r_{2}\right)\right]\right\}\right) .
\end{aligned}
$$

Similarly, (3.15b) and (A3.13) imply that

$$
\hat{h}_{k}^{12}\left(r_{1}, r_{2}\right)=\hat{h}_{k}^{21}\left(r_{2}, r_{1}\right) \text {. }
$$

We have now found useful functional representations of the radial RDCGF, representations we believe to be new. It remains to note that the non-relativistic limit is consistent with the radial RSCGF found in the last section. In fact, using exactly the same methods as were used in the first two papers of this series to ascertain the non-relativistic limit of the radial Dirac-Coulomb wavefunctions and the radial DiracCoulomb Green function we see that for $k=|k| \simeq l$ and for $k=-|k| \simeq-l-1$

$$
\left(\begin{array}{ll}
\hat{g}_{k}^{11} & \hat{g}_{k}^{12} \\
\hat{g}_{k}^{21} & \hat{g}_{k}^{22}
\end{array}\right) \simeq\left(\begin{array}{ll}
\hat{g}_{l} & 0 \\
0 & 0
\end{array}\right) .
$$

\section{Recursion relations for the radial Green functions}

In appendix 3 we prove that

$$
\begin{aligned}
& 2\left[\lambda\left(\frac{\mathrm{d}}{\mathrm{d} r_{1}}+\frac{\lambda+1}{r_{1}}\right)-\omega \nu\right] \hat{g}_{\nu \lambda}\left(r_{1}, r_{2} ; \omega\right) \\
&= \delta\left(r_{1}-r_{2}\right) / \sqrt{r_{1} r_{2}}-\tau_{\lambda}\left(r_{1} r_{2}\right)^{\lambda} \mathrm{e}^{-\left(r_{1}+r_{2}\right) \omega} L_{\nu-\lambda-1}^{2 \lambda+1}\left(2 \omega r_{1}\right) L_{\nu-\lambda-1}^{2 \lambda+1}\left(2 \omega r_{2}\right) \\
&+(2 \omega)^{2 \lambda+1} r_{1}^{\lambda-1} r_{2}^{\lambda} \mathrm{e}^{-\left(r_{1}+r_{2}\right) \omega} \\
& \times\left(\sum_{i=\bar{\nu}}^{\infty} \frac{(i+1) !}{\Gamma(2 \lambda+1+i)(\lambda+1+i-\nu)} L_{i+1}^{2 \lambda-1}\left(2 \omega r_{1}\right) L_{j}^{2 \lambda+1}\left(2 \omega r_{2}\right)\right. \\
&+\sigma_{\lambda}\left(\nu^{2}-\lambda^{2}\right) /(2 \omega)^{2 \lambda+3}\left\{\left(A_{\lambda}+2\right) L_{\nu-\lambda}^{2 \lambda-1}\left(2 \omega r_{1}\right) L_{\nu-\lambda-1}^{2 \lambda+1}\left(2 \omega r_{2}\right)\right. \\
&+\left[(\nu-\lambda+1) L_{\nu-\lambda+1}^{2 \lambda-1}\left(2 \omega r_{1}\right)-(\nu+\lambda-1) L_{\nu-\lambda-1}^{2 \lambda-1}\left(2 \omega r_{1}\right)\right] L_{\nu-\lambda-1}^{2 \lambda+1}\left(2 \omega r_{2}\right) \\
&\left.\left.+L_{\nu-\lambda}^{2 \lambda-1}\left(2 \omega r_{1}\right)\left[(\nu-\lambda) L_{\nu-\lambda}^{2 \lambda+1}\left(2 \omega r_{2}\right)-(\nu+\lambda) L_{\nu-\lambda-2}^{2 \lambda+1}\left(2 \omega r_{2}\right)\right]\right\}\right)
\end{aligned}
$$


and, since $\hat{g}$ is symmetric in $r_{1}$ and $r_{2}$ and the delta function is even,

$$
\begin{aligned}
& 2\left[\lambda\left(\frac{\mathrm{d}}{\mathrm{d} r_{2}}-\frac{\lambda-1}{r_{2}}\right)+\omega \nu\right] g_{\nu \lambda-1}\left(r_{1}, r_{2} ; \omega\right) \\
&=- \delta\left(r_{1}-r_{2}\right) / \sqrt{r_{1} r_{2}}+r_{\lambda-1}\left(r_{1} r_{2}\right)^{\lambda-1} \mathrm{e}^{-\left(r_{1}+r_{2}\right) \omega} L_{\nu-\lambda}^{2 \lambda-1}\left(2 \omega r_{1}\right) L_{\nu-\lambda}^{2 \lambda-1}\left(2 \omega r_{2}\right) \\
&-(2 \omega)^{2 \lambda+1} r_{1}^{\lambda-1} r_{2}^{\lambda} \mathrm{e}^{-\left(r_{1}+r_{2}\right) \omega} \\
& \times\left(\sum_{i=0}^{\infty} \frac{(i+1) !}{\Gamma(2 \lambda+1+i)(\lambda+1+i-\nu)} L_{i+1}^{2 \lambda-1}\left(2 \omega r_{1}\right) L_{i}^{2 \lambda+1}\left(2 \omega r_{2}\right)\right. \\
&+\sigma_{\lambda-1} /(2 \omega)^{2 \lambda+1}\left\{\left(A_{\lambda-1}+2\right) L_{\nu-\lambda}^{2 \lambda-1}\left(2 \omega r_{1}\right) L_{\nu-\lambda-1}^{2 \lambda+1}\left(2 \omega r_{2}\right)\right. \\
&+L_{\nu-\lambda}^{2 \lambda-1}\left(2 \omega r_{1}\right)\left[(\nu-\lambda) L_{\nu-\lambda}^{2 \lambda+1}\left(2 \omega r_{2}\right)-(\nu+\lambda) L_{\nu-\lambda-2}^{2 \lambda+1}\left(2 \omega r_{2}\right)\right] \\
&+ {\left.\left.\left[(\nu-\lambda+1) L_{\nu-\lambda+1}^{2 \lambda-1}\left(2 \omega r_{1}\right)-(\nu+\lambda-1) L_{\nu-\lambda-1}^{2 \lambda-1}\left(2 \omega r_{1}\right)\right] L_{\nu-\lambda-1}^{2 \lambda+1}\left(2 \omega r_{2}\right)\right\}\right) . }
\end{aligned}
$$

If at this point we make the assumption that

$$
\sigma_{\lambda-1} / \sigma_{\lambda}=\tau_{\lambda-1} / \tau_{\lambda}=\left(\nu^{2}-\lambda^{2}\right) /(2 \omega)^{2}
$$

(which is clearly true for the particular values of $\sigma_{\lambda}$ and $\tau_{\lambda}$ relevant to the RSCGF and RDCGF) then we can combine (4.1) and (4.2) to deduce the fundamental recursion relation,

$$
\begin{aligned}
2\left[\lambda\left(\frac{\mathrm{d}}{\mathrm{d} r_{1}}+\frac{\lambda+1}{r_{1}}\right)-\omega \nu\right] \hat{g}_{\nu \lambda}\left(r_{1}, r_{2} ; \omega\right)+2\left[\lambda\left(\frac{\mathrm{d}}{\mathrm{d} r_{2}}-\frac{\lambda-1}{r_{2}}\right)+\omega \nu\right] \hat{g}_{\nu \lambda-1}\left(r_{1}, r_{2} ; \omega\right) \\
=-\tau_{\lambda}\left(r_{1} r_{2}\right)^{\lambda} \mathrm{e}^{-\left(r_{1}+r_{2}\right) \omega} L_{\nu-\lambda-1}^{2 \lambda+1}\left(2 \omega r_{1}\right) L_{\nu-\lambda-1}^{2 \lambda+1}\left(2 \omega r_{2}\right) \\
+\tau_{\lambda-1}\left(r_{1} r_{2}\right)^{\lambda-1} \mathrm{e}^{-\left(r_{1}+r_{2}\right) \omega} L_{\nu-\lambda}^{2 \lambda-1}\left(2 \omega r_{1}\right) L_{\nu-\lambda}^{2 \lambda-1}\left(2 \omega r_{2}\right) \\
+\sigma_{\lambda}\left(\nu^{2}-\lambda^{2}\right) /(2 \omega)^{2}\left[A_{\lambda}-A_{\lambda-1}\right] \\
\\
\times r_{1}^{\lambda-1} r_{2}^{\lambda} \mathrm{e}^{-\left(r_{1}+r_{2}\right) \omega} L_{\nu-\lambda}^{2 \lambda-1}\left(2 \omega r_{1}\right) L_{\nu-\lambda-1}^{2 \lambda+1}\left(2 \omega r_{2}\right) .
\end{aligned}
$$

Two further relations can be readily derived from (4.4). We note first the two recursion relations proven in [1] and restated here as (A3.4) and (A3.9). These two relations imply that

$$
\begin{aligned}
& {\left[\lambda\left(\frac{\mathrm{d}}{\mathrm{d} r}+\frac{\lambda+1}{r}\right)-\omega \nu\right] r^{\lambda-1} \mathrm{e}^{-r \omega} L_{\nu-\lambda}^{2 \lambda-1}(2 \omega r)} \\
& \quad=-2 \omega^{2} r^{\lambda} \mathrm{e}^{-r \omega} L_{\nu-\lambda-1}^{2 \lambda+1}(2 \omega r)+2\left(\lambda^{2} / r-\omega \nu\right) r^{\lambda-1} \mathrm{e}^{-r \omega} L_{\nu-\lambda}^{2 \lambda-1}(2 \omega r)
\end{aligned}
$$

and

$$
\begin{aligned}
& {\left[\lambda\left(\frac{\mathrm{d}}{\mathrm{d} r}-\frac{\lambda-1}{r}\right)+\omega \nu\right] r^{\lambda} \mathrm{e}^{-r \omega} L_{\nu-\lambda-1}^{2 \lambda+1}(2 \omega r)} \\
& \quad=\left(\nu^{2}-\lambda^{2}\right) / 2 r^{\lambda-1} \mathrm{e}^{-r \omega} L_{\nu-\lambda}^{2 \lambda-1}(2 \omega r)+2\left(\omega \nu-\lambda^{2} / r\right) r^{\lambda} \mathrm{e}^{-r \omega} L_{\nu-\lambda-1}^{2 \lambda+1}(2 \omega r) .
\end{aligned}
$$


Now, by virtue of the definition of $\hat{g}$, equation (A1.1), we have

$$
\begin{aligned}
& {\left[\lambda\left(\frac{\mathrm{d}}{\mathrm{d} r_{2}}+\frac{\lambda+1}{r_{2}}\right)-\omega \nu\right]\left[\lambda\left(\frac{\mathrm{d}}{\mathrm{d} r_{2}}-\frac{\lambda-1}{r_{2}}\right)+\omega \nu\right] \hat{g}_{\nu \lambda-1}\left(r_{1}, r_{2} ; \omega\right) } \\
&=\lambda^{2}\left[\frac{\mathrm{d}^{2}}{\mathrm{~d} r_{2}^{2}}+\frac{2}{r_{2}} \frac{\mathrm{d}}{\mathrm{d} r_{2}}-\frac{\lambda(\lambda-1)}{r_{2}^{2}}+\frac{2 \omega \nu}{r_{2}}-\omega^{2}\right] \hat{g}_{\nu \lambda-1}\left(r_{1}, r_{2} ; \omega\right) \\
&+\omega^{2}\left(\lambda^{2}-\nu^{2}\right) \hat{g}_{\nu \lambda-1}\left(r_{1}, r_{2} ; \omega\right) \\
&= \omega^{2}\left(\lambda^{2}-\nu^{2}\right) \hat{g}_{\nu \lambda-1}\left(r_{1}, r_{2} ; \omega\right)-\delta\left(r_{1}-r_{2}\right) / r_{1} r_{2} \\
&+\left(\sigma_{\lambda-1}+\tau_{\lambda-1} / r_{2}\right)\left(r_{1} r_{2}\right)^{\lambda-1} \mathrm{e}^{-\left(r_{1}+r_{2}\right) \omega} L_{\nu-\lambda}^{2 \lambda-1}\left(2 \omega r_{1}\right) L_{\nu-\lambda}^{2 \lambda-1}\left(2 \omega r_{2}\right) .
\end{aligned}
$$

Thus, the fundamental relation (4.4) together with (A3.4), (4.5) and (4.7) implies that

$$
\begin{aligned}
& {\left[\lambda\left(\frac{\mathrm{d}}{\mathrm{d} r_{1}}+\frac{\lambda+1}{r_{1}}\right)-\omega \nu\right]\left[\lambda\left(\frac{\mathrm{d}}{\mathrm{d} r_{2}}+\frac{\lambda+1}{r_{2}}\right)-\omega \nu\right] \hat{g}_{\nu \lambda}\left(r_{1}, r_{2} ; \omega\right) } \\
&+\omega^{2}\left(\lambda^{2}-\nu^{2}\right) \hat{g}_{\nu \lambda-1}\left(r_{1}, r_{2} ; \omega\right) \\
&=\lambda^{2} \delta\left(r_{1}-r_{2}\right) /\left(r_{1} r_{2}\right) \\
&-\left(\lambda^{2} \sigma_{\lambda-1}+\omega \nu \tau_{\lambda-1}\right)\left(r_{1} r_{2}\right)^{\lambda-1} \mathrm{e}^{-\left(r_{1}+r_{2}\right) \omega} L_{\nu-\lambda}^{2 \lambda-1}\left(2 \omega r_{1}\right) L_{\nu-\lambda}^{2 \lambda-1}\left(2 \omega r_{2}\right) \\
&+\frac{1}{4}\left(\nu^{2}-\lambda^{2}\right) \sigma_{\lambda-1}\left(A_{\lambda}-A_{\lambda-1}\right)\left(r_{1} r_{2}\right)^{\lambda-1} \mathrm{e}^{-\left(r_{1}+r_{2}\right) \omega} L_{\nu-\lambda}^{2 \lambda-1}\left(2 \omega r_{1}\right) L_{\nu-\lambda}^{2 \lambda-1}\left(2 \omega r_{2}\right) \\
&-\frac{1}{4}(2 \omega)^{2} \tau_{\lambda-1} \mathrm{e}^{-\left(r_{1}+r_{2}\right) \omega}\left[r_{1}^{\lambda-1} r_{2}^{\lambda} L_{\nu-\lambda}^{2 \lambda-1}\left(2 \omega r_{1}\right) L_{\nu-\lambda-1}^{2 \lambda+1}\left(2 \omega r_{2}\right)\right. \\
&\left.-r_{1}^{\lambda} r_{2}^{\lambda-1} L_{\nu-\lambda-1}^{2 \lambda+1}\left(2 \omega r_{1}\right) L_{\nu-\lambda}^{2 \lambda-1}\left(2 \omega r_{2}\right)\right] .
\end{aligned}
$$

This relatively complicated equation represents a $\lambda$-lowering relation for the $\hat{g}_{\lambda}$. In fact, as we shall soon see, for the particular cases of the radial RSCGF and RDCGF the relation simplifies.

A $\lambda$-raising relation can be derived in an entirely analogous manner, using now (A3.9) and (4.6). We find in this case that

$$
\begin{aligned}
& {\left[\lambda\left(\frac{\mathrm{d}}{\mathrm{d} r_{1}}-\frac{\lambda-1}{r_{1}}\right)+\omega \nu\right]\left[\lambda\left(\frac{\mathrm{d}}{\mathrm{d} r_{2}}-\frac{\lambda-1}{r_{2}}\right)+\omega \nu\right] \hat{g}_{\nu \lambda-1}\left(r_{1}, r_{2} ; \omega\right) } \\
&+ \omega^{2}\left(\lambda^{2}-\nu^{2}\right) \hat{g}_{\nu \lambda}\left(r_{1}, r_{2} ; \omega\right) \\
&= \lambda^{2} \delta\left(r_{1}-r_{2}\right) / r_{1} r_{2} \\
&-\left(\lambda^{2} \sigma_{\lambda}+\omega \nu \tau_{\lambda}\right)\left(r_{1} r_{2}\right)^{\lambda} \mathrm{e}^{-\left(r_{1}+r_{2}\right)} L_{\nu-\lambda-1}^{2 \lambda+1}\left(2 \omega r_{1}\right) L_{\nu-\lambda-1}^{2 \lambda+1}\left(2 \omega r_{2}\right) \\
&-\frac{1}{4} \sigma_{\lambda}\left(\nu^{2}-\lambda^{2}\right)\left(A_{\lambda}-A_{\lambda-1}\right)\left(r_{1} r_{2}\right)^{\lambda} \mathrm{e}^{-\left(r_{1}+r_{2}\right)} L_{\nu-\lambda-1}^{2 \lambda+1}\left(2 \omega r_{1}\right) L_{\nu-\lambda-1}^{2 \lambda+1}\left(2 \omega r_{2}\right) \\
&-\frac{1}{4} \tau_{\lambda}\left(\nu^{2}-\lambda^{2}\right) \mathrm{e}^{-\left(r_{1}+r_{2}\right)}\left[r_{1}^{\lambda-1} r_{2}^{\lambda} L_{\nu-\lambda}^{2 \lambda-1}\left(2 \omega r_{1}\right) L_{\nu-\lambda-1}^{2 \lambda+1}\left(2 \omega r_{2}\right)\right. \\
&\left.+r_{1}^{\lambda} r_{2}^{\lambda-1} L_{\nu-\lambda-1}^{2 \lambda+1}\left(2 \omega r_{1}\right) L_{\nu-\lambda}^{2 \lambda-1}\left(2 \omega r_{2}\right)\right] .
\end{aligned}
$$

The recursion relations given above for the general radial RCGF subject to the condition (4.3) can be applied to the specific functions we are considering in this paper quite easily. All that is required are the particular values of $\sigma_{\lambda}, \tau_{\lambda}$, and $A_{\lambda}$ relevant to the radial RSCGF and RDCGF. In both cases (4.3) holds, and it is a simple matter, given the definitions of the wavefunctions presented in [1], to derive the following formulae. In the non-relativistic case, (4.8) and (4.9) become

$$
\begin{gathered}
\hbar^{2} / 2 m\left[l\left(\frac{\mathrm{d}}{\mathrm{d} r_{1}}+\frac{l+1}{r_{1}}\right)-1 / a\right]\left[l\left(\frac{\mathrm{d}}{\mathrm{d} r_{2}}+\frac{l+1}{r_{2}}\right)-1 / a\right] \hat{g}_{l}\left(r_{1}, r_{2} ; n\right) \\
=\hbar^{2} / 2 m\left(n^{2}-l^{2}\right) /(a n)^{2} \hat{g}_{l-1}\left(r_{1}, r_{2} ; n\right) \\
+l^{2} \delta\left(r_{1}-r_{2}\right) / r_{1} r_{2}-l^{2} R_{n l-1}\left(r_{1}\right) R_{n l-1}\left(r_{2}\right)
\end{gathered}
$$


and

$$
\begin{gathered}
\hbar^{2} / 2 m\left[l\left(\frac{\mathrm{d}}{\mathrm{d} r_{1}}-\frac{l-1}{r_{1}}\right)+1 / a\right]\left[l\left(\frac{\mathrm{d}}{\mathrm{d} r_{1}}-\frac{l-1}{r_{2}}\right)+1 / a\right] \hat{g}_{l-1}\left(r_{1}, r_{2} ; n\right) \\
=\hbar^{2} / 2 m\left(n^{2}-l^{2}\right) /(a n)^{2} \hat{g}_{l}\left(r_{1}, r_{2} ; n\right) \\
+l^{2} \delta\left(r_{1}-r_{2}\right) / r_{1} r_{2}-l^{2} R_{n l}\left(r_{1}\right) R_{n l}\left(r_{2}\right)
\end{gathered}
$$

while the fundamental relation (4.4) becomes

$$
\left[l\left(\frac{\mathrm{d}}{\mathrm{d} r_{1}}+\frac{l+1}{r_{1}}\right)-1 / a\right] \hat{g}_{l}\left(r_{1}, r_{1} ; n\right)=-\left[l\left(\frac{\mathrm{d}}{\mathrm{d} r_{2}}-\frac{l-1}{r_{2}}\right)+1 / a\right] \hat{g}_{l-1}\left(r_{1}, r_{2} ; n\right) .
$$

In the relativistic case we find, in the notation of $[1]$,

$$
\begin{aligned}
\hbar c /\left(\varepsilon_{N \gamma} k+\varepsilon_{0} \gamma\right) & {\left[\gamma\left(\frac{\mathrm{d}}{\mathrm{d} r_{1}}+\frac{\gamma+1}{r_{1}}\right)-\alpha Z \varepsilon_{N \gamma}\right]\left[\gamma\left(\frac{\mathrm{d}}{\mathrm{d} r_{2}}+\frac{\gamma+1}{r_{2}}\right)-\alpha Z \varepsilon_{N \gamma}\right] \hat{h}_{k}^{11}\left(r_{1}, r_{2}\right) } \\
= & -\hbar c \omega_{N \gamma}^{2} N(N+2 \gamma) /\left(\varepsilon_{N \gamma} k-\varepsilon_{0} \gamma\right) \hat{h}_{k}^{22}\left(r_{1}, r_{2}\right)+2 \gamma^{2} /(\kappa+\gamma) \delta\left(r_{1}-r_{2}\right) / r_{1} r_{2} \\
& -2 k g_{2}^{N k}\left(r_{1}\right) g_{2}^{N k}\left(r_{2}\right)-\alpha Z\left[g_{1}^{N k}\left(r_{1}\right) g_{2}^{N k}\left(r_{2}\right)+g_{2}^{N k}\left(r_{1}\right) g_{1}^{N k}\left(r_{2}\right)\right]
\end{aligned}
$$

and

$$
\begin{aligned}
\hbar c /\left(\varepsilon_{N \gamma} k-\varepsilon_{0} \gamma\right) & {\left[\gamma\left(\frac{\mathrm{d}}{\mathrm{d} r_{1}}-\frac{\gamma-1}{r_{\mathrm{t}}}\right)+\alpha Z \varepsilon_{N \gamma}\right]\left[\gamma\left(\frac{\mathrm{d}}{\mathrm{d} r_{2}}-\frac{\gamma-1}{r_{2}}\right)+\alpha Z \varepsilon_{N \gamma}\right] \hat{h}_{k}^{22}\left(r_{1}, r_{2}\right) } \\
= & -\hbar c \omega_{N \gamma}^{2} N(N+2 \gamma) /\left(\varepsilon_{N \gamma} k+\varepsilon_{0} \gamma\right) \hat{h}_{k}^{11}\left(r_{1}, r_{2}\right)+2 \gamma^{2} /(\kappa+\gamma) \delta\left(r_{1}-r_{2}\right) / r_{1} r_{2} \\
& -2 k g_{1}^{N k}\left(r_{1}\right) g_{1}^{N k}\left(r_{2}\right)-\alpha Z\left[g_{1}^{N k}\left(r_{1}\right) g_{2}^{N k}\left(r_{2}\right)+g_{2}^{N k}\left(r_{1}\right) g_{1}^{N k}\left(r_{2}\right)\right] .
\end{aligned}
$$

In the relativistic case the fundamental relation (4.4) is actually a relation between the off-diagonal terms of the radial RDCGF.

The recursion relations for the radial RSCGF (4.10) and (4.11) were given in a slightly different form in [8]. The relations for the relativistic functions are, we believe, new.

\section{Matrix elements of the radial RCGF}

In the previous paper [2] we presented two different techniques which lead directly to formulae for matrix elements of the radial CGF. The first, and most general technique depends on the evaluation of double Laplace transforms (DLTs) of the radial CGF, and allows for the direct computation of a wide range of matrix elements. Though various different forms of the DLT were given, rather complicated analysis would be required for some calculations, especially if the number of terms involved was significant. Compact expressions for a somewhat more restricted class of matrix elements are possible using the second technique, which is basically a generalization of the hypervirial theorem well known in the study of matrix elements of hydrogenic wavefunctions. 
Both techniques become rather less useful when applied to the RCGF, in the first case because we have only presented one form for the radial RCGF (the Sturmian form), and in the second case because the defining differential equation for the radial RCGF contains additional terms.

Nonetheless these difficulties do not often emerge in actual calculations involving the RCGF. Usually we are interested in the matrix elements of the radial RCGF with respect to the corresponding wavefunction. In this case, the energy variable of the RCGF coincides with the eigenvalue of the wavefunction. In such circumstances, concise expressions for the matrix elements may be found by appealing to the orthogonality properties of Laguerre polynomials. For example

$$
\int_{0}^{\infty} \mathrm{e}^{-x} x^{\alpha} L_{i}^{\alpha}(x) L_{j}^{\alpha}(x) \mathrm{d} x=\delta_{i, j} \Gamma(\alpha+i+1) / i !
$$

implies that

$$
\begin{gathered}
\int_{0}^{\infty} \mathrm{e}^{-\omega r_{1}} r_{1}^{\lambda+1} L_{\nu-\lambda-1}^{2 \lambda+1}\left(2 \omega r_{1}\right) \hat{g}_{\nu \lambda}\left(r_{1}, r_{2} ; \omega\right) \mathrm{e}^{-\omega r_{2}} r_{2}^{\lambda+1} L_{\nu-\lambda-1}^{2 \lambda+1}\left(2 \omega r_{2}\right) \mathrm{d} r_{1} \mathrm{~d} r_{2} \\
=\sigma_{\lambda} A_{\lambda} /(2 \omega)^{4 \lambda+6}[\Gamma(\nu+\lambda+1) /(\nu-\lambda-1) !]^{2} .
\end{gathered}
$$

Similarly, since

$$
\int_{0}^{\infty} \mathrm{e}^{-x} x^{\alpha} L_{i}^{\alpha-1}(x) L_{j}^{\alpha+1}(x) \mathrm{d} x= \begin{cases}\alpha \Gamma(\alpha+i) / i ! & j \geqslant i \\ -\Gamma(\alpha+i) /(i-1) ! & j=i-1 \\ 0 & j<i-1\end{cases}
$$

and

${ }_{3} F_{2}\left(\begin{array}{c}a, 1,1 \\ b, 2\end{array} ; 1\right)=(b-1) /(a-1)[\psi(b-1)-\psi(b-a)] \quad a \neq 1, \mathscr{R}(b-a)>0$

we can easily show that

$$
\begin{aligned}
\int_{0}^{\infty} \mathrm{e}^{-\omega r_{1}} r_{1}^{\lambda} L_{\nu-\lambda}^{2 \lambda-1}\left(2 \omega r_{1}\right) \hat{g}_{\nu \lambda}\left(r_{1}, r_{2} ; \omega\right) \mathrm{e}^{-\omega r_{2}} r_{2}^{\lambda} L_{\nu-\lambda}^{2 \lambda-1}\left(2 \omega r_{2}\right) \mathrm{d} r_{1} \mathrm{~d} r_{2} \\
=(2 \omega)^{-2 \lambda-1}[\Gamma(\nu+\lambda) /(\nu-\lambda) !]^{2}\left\{\sigma_{\lambda}(\nu-\lambda)^{2}\left(A_{\lambda}-4 \lambda\right) /(2 \omega)^{2 \lambda+3}\right. \\
\left.+4 \lambda^{2}(\nu-\lambda-1) ! / \Gamma(\nu+\lambda+1)[\psi(\nu+\lambda+1)-\psi(2 \lambda+1)]\right\} .
\end{aligned}
$$

These two integrals are required for the calculation of the relativistic shift in the energy due to a small perturbation of $Z$, the nuclear charge, in hydrogenic ions. Though this calculation is of no intrinsic interest, given that the standard formula for the energy levels is still valid, it nonetheless provides us with a simple test of the validity of many of the results obtained in this paper, and is therefore presented in section 6 .

Âlthough many other such integrais can be derived from the bàsic properties ôf Laguerre polynomials, if all else fails we can resort to the calculation of matrix elements from the double Laplace transforms,

$$
\hat{K}_{\mu_{1} \mu_{2}}^{\nu \lambda}\left(p_{1}, p_{2} ; \omega\right)=\int_{0}^{\infty} r_{1}^{\mu_{1}} \mathrm{e}^{-\omega r_{1} p_{1}} \hat{g}_{\nu \lambda}\left(r_{1}, r_{2} ; \omega\right) r_{2}^{\mu_{2}} \mathrm{e}^{-\omega r_{2} p_{2}} \mathrm{~d} r_{1} \mathrm{~d} r_{2}
$$


The general form for $\hat{K}$, which we omit here, is found as in [2] using the formula

$$
\begin{aligned}
& \int_{0}^{\infty} r^{\mu} \mathrm{e}^{-\omega r p}\left[r^{\lambda} \mathrm{e}^{-\omega r} L_{i}^{2 \lambda+1}(2 \omega r)\right] \mathrm{d} r \\
&=\omega^{-\mu-\lambda-1} \Gamma(\mu+\lambda+i+1) / i !(p-1)^{i}(p+1)^{-i-\mu-\lambda-1} \\
& \times_{2} F_{1}\left(-i, \lambda+1-\mu ;-\mu-\lambda-i ;\left[\frac{p+1}{p-1}\right]\right) .
\end{aligned}
$$

A particular case of the DLT, which has attracted attention elsewhere [13], is obtained when $\mu_{1}=\mu_{2}=\lambda+1$. Then the hypergeometric functions in (5.7) become unity and we find

$$
\begin{aligned}
\hat{K}_{\lambda+1 \lambda+1}^{\nu \lambda}\left(p_{1}, p_{2} ; \omega\right) & =2^{2 \lambda+1} / \omega\left[\left(p_{1}+1\right)\left(p_{2}+1\right)\right]^{-2 \lambda-2} \\
& \times\left(\sigma_{\lambda} /(2 \omega)^{2 \lambda+3}[\Gamma(\nu+\lambda+1) /(\nu-\lambda-1) !]^{2}\left(P_{1} P_{2}\right)^{2-\lambda-1}\right. \\
& \times\left[(\nu+\lambda+1)\left(p_{1}+P_{2}\right)-(\nu-\lambda-1)\left(1 / P_{1}+1 / P_{2}\right)+A_{\lambda}\right] \\
& \left.+\sum_{i=0}^{\infty} \frac{\Gamma(2 \lambda+2+i)}{i !(\lambda+1-\nu+i)}\left(P_{1} P_{2}\right)^{i}\right)
\end{aligned}
$$

where $p_{1}=\left(p_{1}-1\right) /\left(p_{1}+1\right)$ and $P_{2}=\left(p_{2}-1\right) /\left(p_{2}+1\right)$. The infinite sum in this expression can be transformed in such a way as to reduce to a finite sum when $\lambda$ is an integer, as is the case for the radial RSCGF. We begin by noting that

$$
\begin{gathered}
(j-1) ! \sum_{i=0}^{\infty} \frac{\Gamma(\alpha+i+1)}{i !(i+1-j)} x^{i} \\
=\Gamma(\alpha+j)[\psi(j)+\gamma] x^{j-1}+\sum_{i=1}^{\infty} \frac{\Gamma(\alpha+i+j)}{i i !} x^{j+j-1} \\
\quad-\sum_{i=0}^{j-2} i ! \Gamma(\alpha-i+j-1)[x /(1-x)]^{j-2-i} \\
1 / m ! \sum_{i=1}^{\infty} \frac{(m+i) !}{i i !} x^{i}=-\gamma-\log (1-x)-(1-x)^{-m} \sum_{i=0}^{m}\left(\begin{array}{c}
m \\
i
\end{array}\right) \psi(i+1)(-x)^{i}
\end{gathered}
$$

and, for $\beta$ not a positive integer

$1 / \Gamma(\beta+1) \sum_{i=1}^{\infty} \frac{\Gamma(\beta+i+1)}{i i !} x^{i}=\sum_{i=0}^{\infty}(1-x)^{i-\beta} /(\beta-i)-\log x+[\psi(-\beta)-\gamma]$.

where $\gamma=\psi(1)$ is Euler's constant. Thus in the non-relativistic case, when $\lambda=l$ and $\nu=n$ we have

$$
\begin{aligned}
& \sum_{i=0}^{\infty} \frac{(2 l+1+i) !}{i !(l+1-n+i)}\left(P_{1} P_{2}\right)^{i} \\
&=\frac{(n+l) !}{(n-l-1) !}\left\{( P _ { 1 } P _ { 2 } ) ^ { n - 1 - 1 } \left[\psi(n-l)+\log \frac{\left(p_{1}+1\right)\left(p_{2}+1\right)}{2\left(p_{1}+p_{2}\right)}\right.\right. \\
&\left.-\left(\frac{\left(p_{1}+1\right)\left(p_{2}+1\right)}{2\left(p_{1}+p_{2}\right)}\right)^{n+1} \sum_{i=0}^{\infty}\left(\begin{array}{c}
n+l \\
i
\end{array}\right)\left(P_{1} P_{2}\right)^{i}\right] \\
&\left.-\sum_{i=0}^{n-i-2} \frac{i !(n+l-1-i) !}{(n+l) !}\left(\frac{\left(p_{1}-1\right)\left(p_{2}-1\right)}{2\left(p_{1}+p_{2}\right)}\right)^{n-1-2-i}\right\}
\end{aligned}
$$


while in the general case

$$
\begin{aligned}
\sum_{i=0}^{\infty} \frac{\Gamma(2 \lambda+2+i)}{i !(\lambda+1-\nu+i)}\left(P_{1} P_{2}\right)^{i} & \\
= & \frac{\Gamma(\nu+\lambda+1)}{(\nu-\lambda-1) !}\left\{( P _ { 1 } P _ { 2 } ) ^ { \nu - \lambda - 1 } \left[\psi(\nu-\lambda)+\psi(-\nu-\lambda)-\log \left(P_{1} P_{2}\right)\right.\right. \\
& \left.+\sum_{i=0}^{\infty} 1 /(\nu+\lambda-i)\left(\frac{2\left(p_{1}+p_{2}\right)}{\left(p_{1}+1\right)\left(p_{2}+1\right)}\right)^{i-\nu-\lambda}\right] \\
& \left.-\sum_{i=0}^{\nu-\lambda-2} \frac{i ! \Gamma(\nu+\lambda-i)}{\Gamma(\nu+\lambda+1)}\left(\frac{2\left(p_{1}+p_{2}\right)}{\left(p_{1}-1\right)\left(p_{2}-1\right)}\right)^{i-\nu+\lambda+2}\right\} .
\end{aligned}
$$

The non-relativistic case can also be treated by splitting the infinite sum into two parts,

$$
\begin{aligned}
\sum_{i=0}^{\infty} \frac{(2 l+1+i) !}{i !(l+1-} & n+i) \\
= & \sum_{i=0}^{n-l-2} \frac{(2 l+1+i) !}{i !(l+1-n+i)}\left(P_{1} P_{2}\right)^{i} \\
= & \sum_{i=0}^{n-l-2} \frac{(2 l+1+i) !}{i !(l+1-n+i)}\left(P_{1} P_{2}\right)^{i}+\left(P_{1} P_{2}\right)^{n-1-1} \sum_{i=0}^{\infty} \frac{(n+l+1+i) !}{(n-l+i) !(i+1)}\left(P_{1} P_{2}\right)^{i+1} \\
& +1 /(n-l-1) !\left(P_{1} P_{2}\right)^{n-1-1}\left\{(n+l) ! \log \left(\frac{\left(p_{1}+1\right)\left(p_{2}+1\right)}{2\left(p_{1}+p_{2}\right)}\right)\right. \\
& \left.+(2 l+1) ! \sum_{i=0}^{2 l} \frac{(n-l-1+i) !}{i !(2 l+1-i)}\left[\left(\frac{\left(p_{1}+1\right)\left(p_{2}+1\right)}{2\left(p_{1}+p_{2}\right)}\right)^{2 l+1-i}-1\right]\right\}
\end{aligned}
$$

a result which has been derived in a different manner elsewhere [13].

The final method of obtaining matrix elements we will consider here is an extension of the hypervirial theorem which we treated in [2]. As before, we assume we have operators $A_{\nu^{\prime} \lambda^{\prime} \omega^{\prime}}^{\nu \lambda \omega^{\prime}}$ and $B_{\nu^{\prime} \lambda^{\prime} \omega^{\prime}}^{\nu \lambda \omega^{\prime}}$ related by

$$
\begin{gathered}
{\left[\frac{\mathrm{d}^{2}}{\mathrm{~d} r^{2}}+\frac{2}{r} \frac{\mathrm{d}}{\mathrm{d} r}\right.} \\
\left.-\frac{\lambda^{\prime}\left(\lambda^{\prime}+1\right)}{r^{2}}+\frac{2 \omega^{\prime} \nu^{\prime}}{r}-\omega^{\prime 2}\right] A_{\nu^{\prime} \lambda^{\prime} \omega^{\prime}}^{\nu \lambda \omega}\left(r^{\lambda} \mathrm{e}^{-\omega r} L_{\nu-\lambda-1}^{2 \lambda+1}(2 \omega r)\right) \\
=B_{\nu^{\prime} \lambda^{\prime} \omega^{\prime}}^{\nu \lambda \omega}\left(r^{\lambda} \mathrm{e}^{-\omega r} L_{\nu-\lambda-1}^{2 \lambda+1}(2 \omega r)\right)
\end{gathered}
$$

though now both $\nu-\lambda-1$ and $\nu^{\prime}-\lambda^{\prime}-1$ are non-negative integers. The radial $\mathrm{RCGF}$ $\hat{\mathrm{g}}_{\nu \lambda}$ satisfies (A1.1), so it immediately follows that

$$
\begin{aligned}
\int_{0}^{\infty} r_{1}^{2} \hat{g}_{\nu^{\prime} \lambda^{\prime}}\left(r_{1},\right. & \left.r_{2} ; \omega^{\prime}\right) B_{\nu^{\prime} \lambda^{\prime} \omega^{\prime}}^{\nu \lambda \omega}\left(r_{1}^{\lambda} \mathrm{e}^{-\omega r_{1}} L_{\nu-\lambda-1}^{2 \lambda+1}\left(2 \omega r_{1}\right)\right) \mathrm{d} r_{1} \\
= & -A_{\nu^{\prime} \lambda^{\prime} \omega^{\prime}}^{\nu \lambda \omega}\left(r_{2}^{\lambda} \mathrm{e}^{-\omega r_{2}} L_{\nu-\lambda-1}^{2 \lambda+1}\left(2 \omega r_{2}\right)\right) \\
& +\int_{0}^{\infty} r_{1}^{2}\left(\sigma_{\lambda^{\prime}}+\tau_{\lambda^{\prime}} / r_{1}\right)\left(r_{1}^{\lambda^{\prime}} \mathrm{e}^{-\omega^{\prime} r_{1}} L_{\nu^{\prime}-\lambda^{\prime}-1}^{2 \lambda^{\prime}+1}\left(2 \omega r_{1}\right)\right) \\
& \times A_{\nu^{\prime} \lambda^{\prime} \omega^{\prime}}^{\nu \lambda \omega}\left(r_{1}^{\lambda} \mathrm{e}^{-\omega r_{1}} L_{\nu-\lambda-1}^{2 \lambda+1}\left(2 \omega r_{1}\right)\right) \mathrm{d} r_{1}\left(r_{2}^{\lambda^{\prime}} \mathrm{e}^{-\omega^{\prime} r_{2}} L_{\nu^{\prime}-\lambda^{\prime}-1}^{2 \lambda^{\prime}+1}\left(2 \omega r_{2}\right)\right)
\end{aligned}
$$


which may be compared with [2] (6.3). Further reduction is possible if a third operator $C_{\nu^{\prime} \lambda^{\prime} \omega^{\prime}}^{\nu \lambda \omega}$ exists, such that

$$
\begin{gathered}
{\left[\frac{\mathrm{d}^{2}}{\mathrm{~d} r^{2}}+\frac{2}{r} \frac{\mathrm{d}}{\mathrm{d} r}-\frac{\lambda^{\prime}\left(\lambda^{\prime}+1\right)}{r^{2}}+\frac{2 \omega^{\prime} \nu^{\prime}}{r}-\omega^{\prime 2}\right] C_{\nu^{\prime} \lambda^{\prime} \omega^{\prime}}^{\nu \lambda \omega} \cdot\left(r^{\lambda} \mathrm{e}^{-\omega r} L_{\nu-\lambda-1}^{2 \lambda+1}(2 \omega r)\right)} \\
=\left(\sigma_{\lambda^{\prime}}+\tau_{\lambda^{\prime}} / r\right) A_{\nu^{\prime} \lambda^{\prime} \omega}^{\nu \lambda \omega}\left(r^{\lambda} \mathrm{e}^{-\omega r} L_{\nu-\lambda-1}^{2 \lambda+1}(2 \omega r)\right)
\end{gathered}
$$

since, in that case, by the hypervirial theorem for wavefunctions, the integral and thus the second term on the RHS of (5.16) vanishes.

\section{Worked example and conclusions}

Since the Sturmian form of the radial RDCGF is rather complex, we consider it important to test it here by employing it to solve a problem for which the solution is already known. Suppose we perturb the nuclear charge $Z$ by a small amount $\eta$ and calculate the resulting second-order shift in the energy levels. This can be done easily enough by expanding the relativistic energy,

$$
E=m c^{2}(N+\gamma) /\left[(N+\gamma)^{2}+(\alpha Z)^{2}\right]^{1 / 2}
$$

as a Taylor series in $\eta$. The coefficient of $\eta^{2}$, representing the second-order shift, is then given by

$$
\begin{aligned}
\Delta^{2} E_{\text {exact }}=-(\alpha \eta)^{2} m c^{2} /\left(2 \gamma^{3}\right)\left[\left(\kappa^{2}+N \gamma^{3}\right) /\left[N^{2}+\kappa^{2}+2 N \gamma\right]^{3 / 2}\right. \\
\left.+3\left(\kappa^{2}-\gamma^{2}\right) N \gamma\left(\kappa^{2}+N \gamma\right) /\left[N^{2}+\kappa^{2}+2 N \gamma\right]^{5 / 2}\right] .
\end{aligned}
$$

Alternatively, we might try to use second-order perturbation theory, in which case the energy shift is given by

$$
\begin{aligned}
& \Delta^{2} E_{\text {pert }}=-\int\left(\alpha \eta \hbar c / r_{1}\right)\left[\begin{array}{ll}
f_{1}^{N \kappa}\left(r_{1}\right) & f_{2}^{N \kappa}\left(r_{1}\right)
\end{array}\right]\left(\begin{array}{ll}
\hat{g}_{\kappa}^{11}\left(r_{1}, r_{2}\right) & \hat{g}_{\kappa}^{12}\left(r_{1}, r_{2}\right) \\
\hat{g}_{\kappa}^{21}\left(r_{1}, r_{2}\right) & \hat{g}_{\kappa}^{22}\left(r_{1}, r_{2}\right)
\end{array}\right) \\
& \times\left(\begin{array}{l}
f_{1}^{N \kappa}\left(r_{2}\right) \\
f_{2}^{N \kappa}\left(r_{2}\right)
\end{array}\right)\left(\alpha \eta \hbar c / r_{2}\right)\left(r_{1} r_{2}\right)^{2} \mathrm{~d} r_{1} \mathrm{~d} r_{2} .
\end{aligned}
$$

which becomes, written in terms of the transformed radial functions,

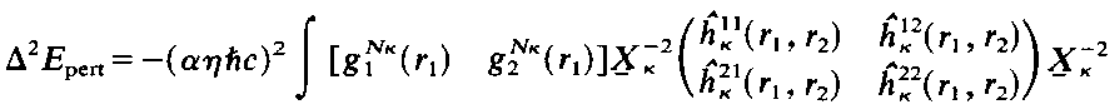

$$
\begin{aligned}
& \times\left(\begin{array}{l}
g_{1}^{N \kappa}\left(r_{2}\right) \\
g_{2}^{N \kappa}\left(r_{2}\right)
\end{array}\right)\left(r_{1} r_{2}\right) \mathrm{d} r_{1} \mathrm{~d} r_{2} .
\end{aligned}
$$

The radial integrals of off-diagonal terms may be simplified by noting that $\hat{h}_{\kappa}^{12}\left(r_{1}, r_{2}\right)=$ $\hat{h}_{\kappa}^{21}\left(r_{2}, r_{1}\right)$. The remaining integrals are performed using (5.1) and (5.3) and integrals given in [1] appendix 2 . Some tedious manipulation of the resulting expressions leads directly to (6.2). Thus we see not only that the results of section 3 are consistent with this calculation, but also that real and exact calculations are possible within the framework of our analysis.

The whole purpose of this series of papers has been the presentation of a method by which exact relativistic calculations of second-order processes may be reduced to simple generalizations of equivalent non-relativistic calculations. In a further paper 
we will present a detailed analysis of the relativistic polarizability of the hydrogen atom. Many other second-order processes may be treated in a similar manner; for example, the calculation of relativistic two-photon decay rates can be seen to reduce to a generalization of the non-relativistic calculation. As far as we are aware, while the non-relativistic decay rates have been treated analytically, the corresponding relativistic calculation has only been performed numerically $[14,15]$. An analytical calculation would serve to clear up discrepancies between these numerical treatments.

While the most significant aspect of the analysis presented in these three papers has been the simplification of calculations of relativistic second-order processes in hydrogen-like atoms, this is by no means all we have achieved. As a consequence of our treatment of the relativistic equations we have brought to light close similarities between the relativistic and non-relativistic functions. We have seen how the wavefunctions, the Coulomb Green functions and the reduced Coulomb Green functions can be treated as specific instances of more general functions, the recursion properties of which we have found in a general manner. The integral properties of these general functions when specialized to the non-relativistic or relativistic cases lead to powerful tools for calculating matrix elements. It is likely that further properties of the general functions exist which, when discovered, will simplify relativistic calculations even further.

\section{Acknowledgments}

Research support by the Natural Sciences and Engineering Research Council of Canada is gratefully acknowledged.

\section{Appendix 1. General solution of the radial RCGF defining equation}

In this appendix we find the solution of a general radial RCGF defining differential equation which allows us to treat both the relativistic and non-relativistic cases in sections 2 and 3 in the same manner. The problem is to solve

$$
\begin{aligned}
\left(\frac{\mathrm{d}^{2}}{\mathrm{~d} r_{1}^{2}}+\frac{2}{r_{1}} \frac{\mathrm{d}}{\mathrm{d} r_{1}}\right. & \left.-\frac{\lambda(\lambda+1)}{r_{1}^{2}}+\frac{2 \omega \nu}{r_{1}}-\omega^{2}\right) \hat{g}_{\nu \lambda}\left(r_{1}, r_{2} ; \omega\right) \\
= & -\delta\left(r_{1}-r_{2}\right) / r_{1} r_{2}+\left(\sigma_{\lambda}+\tau_{\lambda} / r_{1}\right)\left(r_{1} r_{2}\right)^{\lambda} \mathrm{e}^{-\left(r_{1}+r_{2}\right) \omega} \\
& \times L_{\nu-\lambda-1}^{2 \lambda+\mathrm{i}}\left(2 \omega r_{1}\right) L_{\nu-\lambda-1}^{2 \lambda+\mathrm{i}}\left(2 \omega r_{2}\right)
\end{aligned}
$$

where $\nu-\lambda-1$ a positive integer, and where

$$
\nu \sigma_{\lambda} / \omega+\tau_{\lambda}=(2 \omega)^{2 \lambda+2}(\nu-\lambda-1) ! / \Gamma(\nu+\lambda+1)
$$

subject to the conditions that

$$
\begin{aligned}
& \hat{g}_{\nu \lambda}\left(r_{1}, r_{2} ; \omega\right) \rightarrow \mathrm{O}\left(r_{1}^{\lambda}\right) \quad \text { as } r_{1} \rightarrow 0 \\
& \hat{g}_{\nu \lambda}\left(r_{1}, r_{2} ; \omega\right) \rightarrow \mathrm{e}^{-r_{1} \omega} \quad \text { as } \quad r_{1} \rightarrow \infty \\
& \hat{g}_{\nu \lambda}\left(r_{1}, r_{2} ; \omega\right)=\hat{g}_{\nu \lambda}\left(r_{2}, r_{1} ; \omega\right) .
\end{aligned}
$$

Our solution begins with the observations that

$\sigma_{\lambda}+\bar{\tau}_{\lambda} / r=\sigma_{\lambda}(2 \omega r-2 \nu) / 2 \omega r+(2 \omega)^{2 \lambda+2}(\nu-\lambda=1) ! / \Gamma(\nu+\lambda+1) 1 / r$ 
and, appealing to the recursion relation for Laguerre polynomials given in appendix 4, $(2 \omega r-2 \nu) L_{\nu-\lambda-1}^{2 \lambda+1}(2 \omega r)=-\left[(\nu-\lambda) L_{\nu-\lambda}^{2 \lambda+1}(2 \omega r)+(\nu+\lambda) L_{\nu-\lambda-2}^{2 \lambda+1}(2 \omega r)\right]$.

Thus, taking as our starting point the solution to the corresponding defining equation for the general radial CGF ([2], appendix 1) and noting ([1] equation (3.28))

$$
\begin{aligned}
\left(\frac{\mathrm{d}^{2}}{\mathrm{~d} r^{2}}+\frac{2}{r} \frac{\mathrm{d}}{\mathrm{d} r}\right. & \left.-\frac{\lambda(\lambda+1)}{\vec{r}^{2}}+\frac{2 \omega \nu}{r}-\omega^{2}\right) r^{\lambda} \mathrm{e}^{-r \omega} L_{i}^{2 \lambda+1}(2 \omega r) \\
& =-2 \omega(i+\lambda+1-\nu) r^{\lambda-1} \mathrm{e}^{-r \omega} L_{i}^{2 \lambda+1}(2 \omega r)
\end{aligned}
$$

we find

$$
\begin{aligned}
\left(\frac{\mathrm{d}^{2}}{\mathrm{~d} r_{1}^{2}}+\frac{2}{r_{1}} \frac{\mathrm{d}}{\mathrm{d} r_{1}}\right. & \left.-\frac{\lambda(\lambda+1)}{r_{1}^{2}}+\frac{2 \omega \nu}{r_{1}}-\omega^{2}\right)\left((2 \omega)^{2 \lambda+1}\left(r_{1} r_{2}\right)^{\lambda} \mathrm{e}^{-\left(r_{1}+r_{2}\right) \omega}\right. \\
& \left.\times \sum_{i=0}^{\infty} \frac{i !}{\Gamma(2 \lambda+2+i)(\lambda+i+1-\nu)} L_{i}^{2 \lambda+1}\left(2 \omega r_{1}\right) L_{i}^{2 \lambda+1}\left(2 \omega r_{2}\right)\right) \\
= & -(2 \omega)^{2 \lambda+2} r_{1}^{\lambda-1} r_{2}^{\lambda} \mathrm{e}^{-\left(r_{1}+r_{2}\right) \omega} \sum_{i=0}^{\infty} \frac{i !}{\Gamma(2 \lambda+2+i)} L_{i}^{2 \lambda+1}\left(2 \omega r_{1}\right) L_{i}^{2 \lambda+1}\left(2 \omega r_{2}\right) \\
\equiv & -\delta\left(r_{1}-r_{2}\right) / r_{1} r_{2}+\left(\sigma_{\lambda}+\tau_{\lambda} / r_{1}\right)\left(r_{1} r_{2}\right)^{\lambda} \mathrm{e}^{-\left(r_{1}+r_{2}\right) \omega} L_{\nu-\lambda-1}^{2 \lambda+1}\left(2 \omega r_{1}\right) \\
& \times L_{\nu-\lambda-1}^{2 \lambda+1}\left(2 \omega r_{2}\right)+\sigma_{\lambda} / 2 \omega r_{1}^{\lambda-1} r_{2}^{\lambda} \mathrm{e}^{-\left(r_{1}+r_{2}\right) \omega} \\
& \times\left[(\nu-\lambda) L_{\nu-\lambda}^{2 \lambda+1}\left(2 \omega r_{1}\right)+(\nu+\lambda) L_{\nu-\lambda-2}^{2 \lambda+1}\left(2 \omega r_{1}\right)\right] L_{\nu-\lambda-1}^{2 \lambda+1}\left(2 \omega r_{2}\right)
\end{aligned}
$$

where, in the last step we have used our representation of the delta function ([2] equation (A1.19))

$$
\begin{gathered}
\left(r_{1} r_{2}\right)^{\lambda+1 / 2} \mathrm{e}^{-\left(r_{1}+r_{2}\right) \omega} \sum_{i=0}^{\infty} \frac{i !}{\Gamma(2 \lambda+2+i)} L_{i}^{2 \lambda+1}\left(2 \omega r_{1}\right) L_{i}^{2 \lambda+1}\left(2 \omega r_{2}\right) \\
=(1 / 2 \omega)^{2 \lambda+2} \delta\left(r_{1}-r_{2}\right) .
\end{gathered}
$$

Now, since

$$
\begin{aligned}
\left(\frac{\mathrm{d}^{2}}{\mathrm{~d} r^{2}}+\frac{2}{r} \frac{\mathrm{d}}{\mathrm{d} r}-\right. & \left.\frac{\lambda(\lambda+1)}{r^{2}}+\frac{2 \omega \nu}{r}-\omega^{2}\right) \\
& \times\left\{\sigma_{\lambda} /(2 \omega)^{2} r^{\lambda} \mathrm{e}^{-r \omega}\left[(\nu-\lambda) L_{\nu-\lambda}^{2 \lambda+1}(2 \omega r)-(\nu+\lambda) L_{\nu-\lambda-2}^{2 \lambda+1}(2 \omega r)\right]\right\} \\
= & -\sigma_{\lambda} / 2 \omega r^{\lambda-1} \mathrm{e}^{-r \omega}\left[(\nu-\lambda) L_{\nu-\lambda}^{2 \lambda+1}(2 \omega r)+(\nu+\lambda) L_{\nu-\lambda-2}^{2 \lambda+1}(2 \omega r)\right]
\end{aligned}
$$

and, given (A1.5) with $i=\nu-\lambda-1$, we find as a solution to (A1.1) symmetrized with respect to $r_{1}$ and $r_{2}$

$$
\begin{aligned}
\hat{g}_{\nu \lambda}\left(r_{1}, r_{2} ; \omega\right)= & (2 \omega)^{2 \lambda+1}\left(r_{1} r_{2}\right)^{\lambda} \mathrm{e}^{-\left(r_{1}+r_{2}\right) \omega} \\
& \times\left(\sum_{i=0}^{\infty} \frac{i !}{\Gamma(2 \lambda+2+i)(\lambda+i+1-\nu)} L_{i}^{2 \lambda+1}\left(2 \omega r_{1}\right) L_{i}^{2 \lambda+1}\left(2 \omega r_{2}\right)\right. \\
& +\sigma_{\lambda}(1 / 2 \omega)^{2 \lambda+3}\left\{\left[(\nu-\lambda) L_{\nu-\lambda}^{2 \lambda+1}\left(2 \omega r_{1}\right)-(\nu+\lambda)\right.\right. \\
& \left.\times L_{\nu-\lambda-2}^{2 \lambda+1}\left(2 \omega r_{1}\right)\right] L_{\nu-\lambda-1}^{2 \lambda+1}\left(2 \omega r_{2}\right)+A_{\lambda} L_{\nu-\lambda-1}^{2 \lambda+1}\left(2 \omega r_{1}\right) L_{\nu-\lambda-1}^{2 \lambda+1}\left(2 \omega r_{2}\right) \\
& \left.\left.+L_{\nu-\lambda-1}^{2 \lambda+1}\left(2 \omega r_{1}\right)\left[(\nu-\lambda) L_{\nu-\lambda}^{2 \lambda+1}\left(2 \omega r_{2}\right)-(\nu+\lambda) L_{\nu-\lambda-2}^{2 \lambda+1}\left(2 \omega r_{2}\right)\right]\right\}\right)
\end{aligned}
$$

where $A_{\lambda}$ is an arbitrary constant, to be determined by the normalization conditions. 


\section{Appendix 2. The normalization integral}

In this appendix we calculate the general normalization integral given by

$$
\begin{aligned}
I_{\lambda}=\int_{0}^{\infty}\left(\sigma_{\lambda}+\right. & \left.\tau_{\lambda} / r_{1}\right) r_{1}^{\lambda} \mathrm{e}^{-\omega r_{1}} L_{\nu-\lambda-1}^{2 \lambda+1}\left(2 \omega r_{1}\right) \hat{g}_{\nu \lambda}\left(r_{1}, r_{2} ; \omega\right) r_{1}^{2} \mathrm{~d} r_{1} \\
= & \int_{0}^{\infty} r_{1}^{\lambda+1} \mathrm{e}^{-\omega r_{1}} \hat{g}_{\nu \lambda}\left(r_{1}, r_{2} ; \omega\right)\left[\left(\sigma_{\lambda} r_{1}+\tau_{\lambda}\right) L_{\nu-\lambda-1}^{2 \lambda+1}\left(2 \omega r_{1}\right)\right] \mathrm{d} r_{1} \\
= & \int_{0}^{\infty} r_{1}^{\lambda+1} \mathrm{e}^{-\omega r_{1}} \hat{g}_{\nu \lambda}\left(r_{1}, r_{2} ; \omega\right) \times\left[-\sigma_{\lambda}(\nu-\lambda) / 2 \omega L_{\nu-\lambda}^{2 \lambda+1}\left(2 \omega r_{1}\right)\right. \\
& \left.+\left(\nu \sigma_{\lambda} / \omega+\tau_{\lambda}\right) L_{\nu-\lambda-1}^{2 \lambda+1}\left(2 \omega r_{1}\right)-\sigma_{\lambda}(\nu+\lambda) / 2 \omega L_{\nu-\lambda-2}^{2 \lambda+1}\left(2 \omega r_{1}\right)\right] \mathrm{d} r_{1}
\end{aligned}
$$

since (see appendix 4)

$$
x L_{\nu-\lambda-1}^{2 \lambda+1}(x)=-(\nu-\lambda) L_{\nu-\lambda}^{2 \lambda+1}(x)+2 \nu L_{\nu-\lambda-1}^{2 \lambda+1}(x)-(\nu+\lambda) L_{\nu-\lambda-2}^{2 \lambda+1}(x) .
$$

Now, taking note of the form of $\hat{\mathrm{g}}_{\nu \lambda}$ given by (A1.2) and the orthogonality property of Laguerre polynomials,

$$
\int_{0}^{\infty} \mathrm{e}^{-x} x^{\alpha} L_{n}^{\alpha}(x) L_{m}^{\alpha}(x) \mathrm{d} x=\delta_{n, m} \Gamma(n+\alpha+1) / n !
$$

we deduce that

$$
\begin{aligned}
& I_{\lambda}=r_{2}^{\lambda} \mathrm{e}^{-\omega r_{2}} L_{\nu-\lambda-1}^{2 \lambda+1}\left(2 \omega r_{2}\right) \sigma_{\lambda} /(2 \omega)^{2} \\
& \times\left[A_{\lambda}-2 \sigma_{\lambda} \nu /(2 \omega)^{2 \lambda+3} \Gamma(\nu+\lambda+1) /(\nu-\lambda-1) !\right]
\end{aligned}
$$

where we have used $(\mathrm{A} 1.2)$ to eliminate $\tau_{\lambda}$ from the RHS.

\section{Appendix 3. Single variable differential properties}

In this appendix we examine $\left[\lambda\left(\mathrm{d} / \mathrm{d} r_{1}+(\lambda+1) / r_{1}\right)-\omega \nu\right] \hat{g}_{\nu \lambda}\left(r_{1}, r_{2} ; \omega\right)$ and $\left[\lambda\left(\mathrm{d} / \mathrm{d} r_{1}-\right.\right.$ $\left.\left.(\lambda-1) / r_{1}\right)+\omega \nu\right] \hat{g}_{\nu \lambda-1}\left(r_{1}, r_{2} ; \omega\right)$. Just as in the case of the ordinary radial CGF [2] knowledge of these two expressions is required for the calculation of the off-diagonal terms of the radial RDCGF (section 3 ), as well as for the derivation of various recursion relations (section 4). We follow closely the method of [2] (appendix 2) although the analysis is here complicated by the presence of additional terms in the general radial RCGF. To facilitate the presentation we begin by splitting the radial RCGF into two parts,

$$
\begin{aligned}
\hat{\mathrm{g}}_{\nu \lambda}^{1}\left(r_{1}, r_{2} ; \omega\right)= & (2 \omega)^{2 \lambda+1}\left(r_{1} r_{2}\right)^{\lambda} \mathrm{e}^{-\left(r_{1}+r_{2}\right) \omega} \\
& \times \sum_{i=0}^{\infty} \frac{i !}{\Gamma(2 \lambda+2+i)(\lambda+i+1-\nu)} L_{i}^{2 \lambda+1}\left(2 \omega r_{1}\right) L_{i}^{2 \lambda+1}\left(2 \omega r_{2}\right) \\
\hat{g}_{\nu \lambda}^{2}\left(r_{1}, r_{2} ; \omega\right)= & \left(r_{1} r_{2}\right)^{\lambda} \mathrm{e}^{-\left(r_{1}+r_{2}\right) \omega} \sigma_{\lambda}(1 / 2 \omega)^{2} \\
& \times\left\{\left[(\nu-\lambda) L_{\nu-\lambda}^{2 \lambda+1}\left(2 \omega r_{1}\right)-(\nu+\lambda) L_{\nu-\lambda-2}^{2 \lambda+1}\left(2 \omega r_{1}\right)\right] L_{\nu-\lambda-1}^{2 \lambda+1}\left(2 \omega r_{2}\right)\right. \\
& +A_{\lambda} L_{\nu-\lambda-1}^{2 \lambda+1}\left(2 \omega r_{1}\right) L_{\nu-\lambda-1}^{2 \lambda+1}\left(2 \omega r_{2}\right) \\
& \left.+L_{\nu-\lambda-1}^{2 \lambda+1}\left(2 \omega r_{1}\right)\left[(\nu-\lambda) L_{\nu-\lambda}^{2 \lambda+1}\left(2 \omega r_{2}\right)-(\nu+\lambda) L_{\nu-\lambda-2}^{2 \lambda+1}\left(2 \omega r_{2}\right)\right]\right\}
\end{aligned}
$$


so that

$$
\hat{g}_{\nu_{\lambda}}\left(r_{1}, r_{2} ; \omega\right)=\hat{g}_{\nu \lambda}^{\mathrm{l}}\left(r_{1}, r_{2} ; \omega\right)+\hat{g}_{\nu \lambda}^{2}\left(r_{1}, r_{2} ; \omega\right) .
$$

Now since ([1] equation (3.29))

$$
\begin{aligned}
& {\left[\lambda\left(\frac{\mathrm{d}}{\mathrm{d} r}+\frac{\lambda+1}{r}\right)-\omega \nu\right]\left[r^{\lambda} \mathrm{e}^{-r \omega} L_{i}^{2 \lambda+1}(2 \omega r)\right] } \\
&= \omega(i+\lambda+1-\nu) r^{\lambda} \mathrm{e}^{-r \omega} L_{i}^{2 \lambda+1}(2 \omega r) \\
&+(i+1)(2 \lambda+i+1) / 2 r^{\lambda-1} \mathrm{e}^{-r \omega} L_{i+1}^{2 \lambda-1}(2 \omega r)
\end{aligned}
$$

we find

$$
\begin{aligned}
& {\left[\lambda\left(\frac{\mathrm{d}}{\mathrm{d} r_{1}}+\frac{\lambda+1}{r_{1}}\right)-\omega \nu\right] \hat{g}_{\nu \lambda}^{1}\left(r_{1}, r_{2} ; \omega\right) } \\
&=(2 \omega)^{2 \lambda+1}\left(r_{1} r_{2}\right)^{\lambda} \mathrm{e}^{-\left(r_{1}+r_{2}\right) \omega}\left(\omega \sum_{i=0}^{\infty} \frac{i !}{\Gamma(2 \lambda+2+i)} L_{i}^{2 \lambda+1}\left(2 \omega r_{1}\right) L_{i}^{2 \lambda+1}\left(2 \omega r_{2}\right)\right. \\
&\left.+1 /\left(2 r_{1}\right) \sum_{i=0}^{\infty} \frac{(i+1) !}{\Gamma(2 \lambda+1+i)(\lambda+1+i-\nu)} L_{i+1}^{2 \lambda-1}\left(2 \omega r_{1}\right) L_{i}^{2 \lambda+1}\left(2 \omega r_{2}\right)\right) \\
&= \frac{1}{2} \delta\left(r_{1}-r_{2}\right) / \sqrt{r_{1} r_{2}} \\
&-\frac{1}{2}\left(\nu \sigma_{\lambda} / \omega+\tau_{\lambda}\right)\left(r_{1} r_{2}\right)^{\lambda} \mathrm{e}^{-\left(r_{1}+r_{2}\right) \omega} L_{\nu-\lambda-1}^{2 \lambda+1}\left(2 \omega r_{1}\right) L_{\nu-\lambda-1}^{2 \lambda+1}\left(2 \omega r_{2}\right) \\
&+\frac{1}{2}(2 \omega)^{2 \lambda+1} r_{1}^{\lambda-1} r_{2}^{\lambda} \mathrm{e}^{-\left(r_{1}+r_{2}\right) \omega} \\
& \times \sum_{i=0}^{\infty} \frac{(i+1) !}{\Gamma(2 \lambda+1+i)(\lambda+1+i-\nu)} L_{i+1}^{2 \lambda-1}\left(2 \omega r_{1}\right) L_{i}^{2 \lambda+1}\left(2 \omega r_{2}\right)
\end{aligned}
$$

where we have employed the representation of the delta function given in ([2] equation (A1.19)) as well as the special relationship between $\sigma_{\lambda}$ and $\tau_{\lambda}$ given in (A1.3). We also find, from (A3.4)

$$
\begin{aligned}
& {\left[\lambda\left(\frac{\mathrm{d}}{\mathrm{d} r_{1}}+\frac{\lambda+1}{r_{1}}\right)-\omega \nu\right] \hat{g}_{\nu \lambda}^{2}\left(r_{1}, r_{2} ; \omega\right) } \\
&=\sigma_{\lambda} /(2 \omega)^{2}\left(r_{1} r_{2}\right) \mathrm{e}^{-\left(r_{1}+r_{2}\right) \omega}\left[1 /\left(2 r_{1}\right)\right. \\
& \times\left\{\left[(\nu-\lambda)(\nu-\lambda+1)(\nu+\lambda+1) L_{\nu-\lambda+1}^{2 \lambda-1}\left(2 \omega r_{1}\right)\right.\right. \\
&\left.-(\nu+\lambda)(\nu-\lambda-1)(\nu+\lambda-1) L_{\nu-\lambda-1}^{2 \lambda-1}\left(2 \omega r_{1}\right)\right] L_{\nu-\lambda-1}^{2 \lambda+1}\left(2 \omega r_{2}\right) \\
&+(\nu-\lambda)(\nu+\lambda) A_{\lambda} L_{\nu-\lambda}^{2 \lambda-1}\left(2 \omega r_{1}\right) L_{\nu-\lambda-1}^{2 \lambda+1}\left(2 \omega r_{2}\right) \\
&\left.+(\nu-\lambda)(\nu+\lambda) L_{\nu-\lambda}^{2 \lambda-1}\left(2 \omega r_{1}\right)\left[(\nu-\lambda) L_{\nu-\lambda}^{2 \lambda+1}\left(2 \omega r_{2}\right)-(\nu+\lambda) L_{\nu-\lambda-2}^{2 \lambda+1}\left(2 \omega r_{2}\right)\right]\right\} \\
&+\omega\left[(\nu-\lambda) L_{\nu-\lambda}^{2 \lambda+1}\left(2 \omega r_{1}\right)+(\nu+\lambda) L_{\nu-\lambda-2}^{2 \lambda+1}\left(2 \omega r_{1}\right)\right] L_{\nu-\lambda-1}^{2 \lambda+1}\left(2 \omega r_{2}\right) \rrbracket .
\end{aligned}
$$

Now

$$
\begin{aligned}
& {\left[(\nu-\lambda) L_{\nu-\lambda}^{2 \lambda+1}(2 \omega r)+(\nu+\lambda) L_{\nu-\lambda-2}^{2 \lambda+1}(2 \omega r)\right]=2(\nu-\omega r) L_{\nu-\lambda-1}^{2 \lambda+1}(2 \omega r)} \\
& =2 \nu L_{\nu-\lambda-1}^{2 \lambda+1}(2 \omega r)-1 /(2 r)\left[(\nu+\lambda)(\nu+\lambda-1) L_{\nu-\lambda-1}^{2 \lambda-1}(2 \omega r)\right. \\
& \left.\quad-2(\nu-\lambda)(\nu+\lambda) L_{\nu-\lambda}^{2 \lambda-1}(2 \omega r)+(\nu-\lambda)(\nu-\lambda+1) L_{\nu-\lambda+1}^{2 \lambda-1}(2 \omega r)\right]
\end{aligned}
$$


and thus, combining (A3.5) and (A3.6)

$$
\begin{aligned}
& 2\left[\lambda\left(\frac{\mathrm{d}}{\mathrm{d} r_{1}}+\frac{\lambda+1}{r_{1}}\right)-\omega \nu\right] \hat{g}_{\nu \lambda}\left(r_{1}, r_{2} ; \omega\right) \\
&= \delta\left(r_{1}-r_{2}\right) / \sqrt{r_{1} r_{2}}-\tau_{\lambda}\left(r_{1} r_{2}\right)^{\lambda} \mathrm{e}^{-\left(r_{1}+r_{2}\right) \omega} L_{\nu-\lambda-1}^{2 \lambda+1}\left(2 \omega r_{1}\right) L_{\nu-\lambda-1}^{2 \lambda+1}\left(2 \omega r_{2}\right) \\
&+(2 \omega)^{2 \lambda+1} r_{1}^{\lambda-1} r_{2}^{\lambda} \mathrm{e}^{-\left(r_{1}+r_{2}\right) \omega} \\
& \times\left(\sum_{i=0}^{\infty} \frac{(i+1) !}{\Gamma(2 \lambda+1+i)(\lambda+1+i-\nu)} L_{i+1}^{2 \lambda-1}\left(2 \omega r_{1}\right) L_{j}^{2 \lambda+1}\left(2 \omega r_{2}\right)\right. \\
&+\sigma_{\lambda}\left(\nu^{2}-\lambda^{2}\right) /(2 \omega)^{2 \lambda+3}\left\{\left(A_{\lambda}+2\right) L_{\nu-\lambda}^{2 \lambda-1}\left(2 \omega r_{1}\right) L_{\nu-\lambda-1}^{2 \lambda+1}\left(2 \omega r_{2}\right)\right. \\
&+\left[(\nu-\lambda+1) L_{\nu-\lambda+1}^{2 \lambda-1}\left(2 \omega r_{1}\right)-(\nu+\lambda-1) L_{\nu-\lambda-1}^{2 \lambda-1}\left(2 \omega r_{1}\right)\right] L_{\nu-\lambda-1}^{2 \lambda+1}\left(2 \omega r_{2}\right) \\
&\left.\left.+L_{\nu-\lambda}^{2 \lambda-1}\left(2 \omega r_{1}\right)\left[(\nu-\lambda) L_{\nu-\lambda}^{2 \lambda+1}\left(2 \omega r_{2}\right)-(\nu+\lambda) L_{\nu-\lambda-2}^{2 \lambda+1}\left(2 \omega r_{2}\right)\right]\right\}\right) .
\end{aligned}
$$

This constitutes our first main result of this appendix.

The second calculation is performed in a similar manner. Since ([1] equation (3.30))

$$
\begin{aligned}
& {\left[\lambda\left(\frac{\mathrm{d}}{\mathrm{d} r}-\frac{\lambda-1}{r}\right)+\omega \nu\right]\left(r^{\lambda-1} \mathrm{e}^{-r \omega} L_{i}^{2 \lambda-1}(2 \omega r)\right)} \\
& \quad=-\omega(i+\lambda-\nu) r^{\lambda-1} \mathrm{e}^{-r \omega} L_{i}^{2 \lambda-1}(2 \omega r)-2 \omega^{2}\left\{1-\delta_{i 0}\right\} r^{\lambda} \mathrm{e}^{-r \omega} L_{i-1}^{2 \lambda+1}(2 \omega r)
\end{aligned}
$$

we now deduce that

$$
\begin{aligned}
{\left[\lambda\left(\frac{\mathrm{d}}{\mathrm{d} r_{1}}-\frac{\lambda-1}{r_{1}}\right)+\omega \nu\right] g_{\nu \lambda-1}^{1}\left(r_{1}, r_{2} ; \omega\right) } \\
=-\frac{1}{2} \delta\left(r_{1}-r_{2}\right) / \sqrt{r_{1} r_{2}}+\frac{1}{2}\left(\nu \sigma_{\lambda-1} / \omega+\tau_{\lambda-1}\right)\left(r_{1} r_{2}\right)^{\lambda-1} \mathrm{e}^{-\left(r_{1}+r_{2}\right) \omega} \\
\quad \times L_{\nu-\lambda}^{2 \lambda-1}\left(2 \omega r_{1}\right) L_{\nu-\lambda}^{2 \lambda-1}\left(2 \omega r_{2}\right)-\frac{1}{2}(2 \omega)^{2 \lambda+1} r_{1}^{\lambda} r_{2}^{\lambda-1} \mathrm{e}^{-\left(r_{1}+r_{2}\right) \omega} \\
\\
\quad \times \sum_{i=0}^{\infty} \frac{(i+1) !}{\Gamma(2 \lambda+1+i)(\lambda+1+i-\nu)} L_{i}^{2 \lambda+1}\left(2 \omega r_{1}\right) L_{i+1}^{2 \lambda-1}\left(2 \omega r_{2}\right)
\end{aligned}
$$

and

$$
\begin{aligned}
{\left[\lambda\left(\frac{\mathrm{d}}{\mathrm{d} r_{1}}-\frac{\lambda-1}{r_{1}}\right)\right.} & +\omega \nu] g_{\nu \lambda-1}^{2}\left(r_{1}, r_{2} ; \omega\right) \\
= & -1 /(2 \omega)^{2} \sigma_{\lambda-1} \omega\left(r_{1} r_{2}\right)^{\lambda-1} \mathrm{e}^{-\left(r_{1}+r_{2}\right) \omega} \\
& \times \llbracket\left[(\nu-\lambda+1) L_{\nu-\lambda+1}^{2 \lambda-1}\left(2 \omega r_{1}\right)+(\nu+\lambda-1) L_{\nu-\lambda-1}^{2 \lambda-1}\left(2 \omega r_{1}\right)\right] L_{\nu-\lambda}^{2 \lambda-1}\left(2 \omega r_{2}\right) \\
& +2 \omega r\left\{\left[(\nu-\lambda+1) L_{\nu-\lambda}^{2 \lambda+1}\left(2 \omega r_{1}\right)-(\nu+\lambda-1) L_{\nu-\lambda-2}^{2 \lambda+1}\left(2 \omega r_{1}\right)\right] L_{\nu-\lambda}^{2 \lambda-1}\left(2 \omega r_{2}\right)\right. \\
& +A_{\lambda-1} L_{\nu-\lambda-1}^{2 \lambda+1}\left(2 \omega r_{1}\right) L_{\nu-\lambda}^{2 \lambda-1}\left(2 \omega r_{2}\right)+L_{\nu-\lambda-1}^{2 \lambda+1}\left(2 \omega r_{1}\right) \\
& \left.\times\left[(\nu-\lambda+1) L_{\nu-\lambda+1}^{2 \lambda-1}\left(2 \omega r_{2}\right)-(\nu+\lambda-1) L_{\nu-\lambda-1}^{2 \lambda-1}\left(2 \omega r_{2}\right)\right]\right\} \rrbracket .
\end{aligned}
$$

Since

$$
\begin{aligned}
(\nu-\lambda+1) & L_{\nu-\lambda+1}^{2 \lambda-1}(2 \omega r)+(\nu+\lambda-1) L_{\nu-\lambda-1}^{2 \lambda-1}(2 \omega r) \\
& =2(\nu-\omega r) L_{\nu-\lambda}^{2 \lambda-1}(2 \omega r) \\
& =2 \nu L_{\nu-\lambda}^{2 \lambda-1}(2 \omega r)-2 \omega r\left[L_{\nu^{\prime}-\lambda}^{2 \lambda+1}(2 \omega r)-2 L_{\nu-\lambda-1}^{2 \lambda+1}(2 \omega r)+L_{\nu-\lambda-2}^{2 \lambda+1}(2 \omega r)\right]
\end{aligned}
$$


we can combine (A3.10) and (A3.11), and thus find

$$
\begin{aligned}
& {\left[\lambda\left(\frac{\mathrm{d}}{\mathrm{d} r_{1}}-\frac{\lambda-1}{r_{2}}\right)+\omega \nu\right] g_{\nu \lambda-1}\left(r_{1}, r_{2} ; \omega\right) } \\
&=-\frac{1}{2} \delta\left(r_{1}-r_{2}\right) / \sqrt{r_{1} r_{2}}+\frac{1}{2} \tau_{\lambda-1}\left(r_{1} r_{2}\right)^{\lambda-1} \mathrm{e}^{-\left(r_{1}+r_{2}\right) \omega} L_{\nu-\lambda}^{2 \lambda-1}\left(2 \omega r_{1}\right) L_{\nu-\lambda}^{2 \lambda-1}\left(2 \omega r_{2}\right) \\
&-\frac{1}{2}(2 \omega)^{2 \lambda+1} r_{1}^{\lambda} r_{2}^{\lambda-1} \mathrm{e}^{-\left(r_{1}+r_{2}\right) \omega} \\
& \times\left(\sum_{i=0}^{\infty} \frac{(i+1) !}{\Gamma(2 \lambda+1+i)(\lambda+1+i-\nu)} L_{i}^{2 \lambda+1}\left(2 \omega r_{1}\right) L_{i+1}^{2 \lambda-1}\left(2 \omega r_{2}\right)\right. \\
&+\sigma_{\lambda-1} /(2 \omega)^{2 \lambda+1}\left\{\left[(\nu-\lambda) L_{\nu-\lambda}^{2 \lambda+1}\left(2 \omega r_{1}\right)-(\nu+\lambda) L_{\nu-\lambda-2}^{2 \lambda+1}\left(2 \omega r_{1}\right)\right] L_{\nu-\lambda}^{2 \lambda-1}\left(2 \omega r_{2}\right)\right. \\
&+\left(2+A_{\lambda-1}\right) L_{\nu-\lambda-1}^{2 \lambda+1}\left(2 \omega r_{1}\right) L_{\nu-\lambda}^{2 \lambda-1}\left(2 \omega r_{2}\right)+L_{\nu-\lambda-1}^{2 \lambda+1}\left(2 \omega r_{1}\right) \\
&\left.\left.\times\left[(\nu-\lambda+1) L_{\nu-\lambda+1}^{2 \lambda-1}\left(2 \omega r_{2}\right)-(\nu+\lambda-1) L_{\nu-\lambda-1}^{2 \lambda-1}\left(2 \omega r_{2}\right)\right]\right\}\right)
\end{aligned}
$$

which constitutes the second result we wished to present in this appendix.

\section{Appendix 4. Notation and special functions}

Hypergeometric functions.

$$
\begin{aligned}
& (\alpha)_{n}=\Gamma(\alpha+n) / \Gamma(\alpha) \\
& { }_{1} F_{1}(\alpha ; \beta ; x)=\sum \frac{(\alpha)_{n}}{(\beta)_{n}} \frac{x^{n}}{n !} \\
& { }_{2} F_{1}(\alpha, \beta ; \gamma ; x)=\sum \frac{(\alpha)_{n}(\beta)_{n}}{(\gamma)_{n}} \frac{x^{n}}{n !} \\
& { }_{p} F_{q}\left(\alpha_{1}, \ldots ; \beta_{1}, \ldots ; x\right)=\sum \frac{\left(\alpha_{1}\right)_{n} \ldots\left(\alpha_{p}\right)_{n}}{\left(\beta_{1}\right)_{n} \ldots\left(\beta_{q}\right)_{n}} \frac{x^{n}}{n !} .
\end{aligned}
$$

Laguerre polynomials.

$$
\begin{aligned}
& L_{n}^{\alpha}(x)=\frac{\Gamma(\alpha+1+n)}{\Gamma(\alpha+1) n !}, F_{1}(-n ; \alpha+1 ; x) \\
& (n+1) L_{n+1}^{\alpha}(x)+(x-\alpha-2 n-1) L_{n}^{\alpha}(x)+(n+\alpha) L_{n-1}^{\alpha}(x)=0
\end{aligned}
$$

Dirac matrices.

$$
\begin{aligned}
\boldsymbol{\alpha} & =\left(\begin{array}{ll}
0 & \boldsymbol{\sigma} \\
\boldsymbol{\sigma} & 0
\end{array}\right) \quad \beta=\left(\begin{array}{rr}
1 & 0 \\
0 & -1
\end{array}\right) \\
\boldsymbol{\sigma} & =\left(\left(\begin{array}{ll}
0 & 1 \\
1 & 0
\end{array}\right),\left(\begin{array}{rr}
0 & -i \\
i & 0
\end{array}\right),\left(\begin{array}{rr}
1 & 0 \\
0 & -1
\end{array}\right)\right) \\
\mathbf{\Sigma} & =\left(\begin{array}{ll}
\boldsymbol{\sigma} & 0 \\
0 & \boldsymbol{\sigma}
\end{array}\right) .
\end{aligned}
$$




\section{References}

[1] Swainson R A and Drake G W F 1991 J. Phys. A: Math. Gen. 2479

[2] Swainson R A and Drake G W F 1991 J. Phys. A: Math. Gen. 2495

[3] Hostler L C 1969 Phys. Rev. 178126

[4] Hameka H F 1967 J. Chem. Phys. 472728

[5] Hostler L 1970 J. Math. Phys. 112966

[6] Sherstyuk A I 1971 Opt. Spectrosc. 30191

[7] Khristenko S V and Vetchinkin S 11971 Opt. Spectrosc. 31269

[8] Johnson B R and Hirschfelder J O 1979 J. Math. Phys. 202484

[9] Zon B A et al. 1972 Sov. J. Nucl. Phys. 15282

[10] Manakov N L and Zapryagaev S A 1976 Phys. Lett. 58A 23

[11] Hylton D J 1984 J. Math. Phys. 251125

[12] Edmonds A R 1957 Angular Momentum in Quantum Mechanics (Princeton, NJ: Princeton University Press)

[13] Hill R N and Huxtable B D 1982 J. Math. Phys. 232365

[14] Goldman S P and Drake G W F 1981 Phys. Rev. A 24183

[15] Parpia F A and Johnson W R 1982 Phys. Rev. A 261142 